\title{
Self-organization of charged particles in circular geometry
}

\author{
R.G. Nazmitdinov, ${ }^{1,2}$ A. Puente, ${ }^{1}$ M. Cerkaski, ${ }^{3}$ and M. Pons ${ }^{1}$ \\ 1 Departament de Física, Universitat de les Illes Balears, E-07122 Palma de Mallorca, Spain \\ ${ }^{2}$ Bogoliubov Laboratory of Theoretical Physics, Joint Institute for Nuclear Research, 141980 Dubna, Russia \\ ${ }^{3}$ Department of Theory of Structure of Matter, Institute of Nuclear Physics PAN, 31-342 Cracow, Poland
}

(Dated: August 16, 2018)

\begin{abstract}
The basic principles of self-organization of one-component charged particles, confined in disk and circular parabolic potentials, are proposed. A system of equations is derived, that allows us to determine equilibrium configurations for an arbitrary, but finite, number of charged particles that are distributed over several rings. Our approach reduces significantly the computational effort in minimizing the energy of equilibrium configurations and demonstrates a remarkable agreement with the values provided by molecular dynamics calculations. With the increase of particle number $n>180$ we find a steady formation of a centered hexagonal lattice that smoothly transforms to valence circular rings in the ground state configurations for both potentials.
\end{abstract}

PACS numbers: $64.70 . \mathrm{kp}, 64.75 . \mathrm{Yz}, 02.20 . \mathrm{Rt}$

\section{INTRODUCTION}

There is an enormous interest in mesoscopic systems consisting of a finite number of interacting particles in a confined geometry. It is well understood that various phenomena, that are suppressed in a continuous limit, are brought about by finiteness and boundaries of these systems [1]. Progress in modern technology allows us to study such phenomena on the same scale, from Bose condensate with some thousand atoms to quantum dots with a few electrons, providing rich information about specific features of correlation effects in mesoscopic systems (see, for example, Ref. 2]). Nowadays, many ideas and concepts introduced, in particular, in condensed matter physics can be realized and analyzed with high accuracy as a function of particle number and boundary properties.

Long ago Wigner predicted that electrons interacting by means of Coulomb forces could create a crystallized structure in a three-dimensional (3D) space at low enough densities and temperatures 3 . At these conditions the potential energy dominates over the kinetic energy and defines equilibrium configurations of electronic systems. This prediction initiated various research lines in diverse branches of physics and chemistry. In particular, the so called Coulomb clusters that result from harmonic confinement of charged particles in two and three dimensions attracted intensive attention, since they are relevant for the description of cold ions in various traps, dusty plasmas, and many other systems.

At moderate number of particles $\left(\sim 10^{3}\right)$ the properties of spherical Coulomb systems may be analysed in terms of simple shell models, in which the constituting particles create concentric spherical surfaces called shells (see Ref.4 and references therein). The crystallization of a one-component plasma for a system size up to $10^{5}$ ions, confined by a spherically symmetric parabolic potential, induced by their mutual Coulomb interaction, has been studied by means of molecular dynamics (MD) simulations [5]. It was found that the formation of the bcc lattice provides better ground state energies than shell configurations for a number of ions $n \geq 2 \times 10^{4}$. Signatures of Wigner crystallization were also observed in two-dimensional (2D) distributions of electrons on the surface of liquid helium [6. A phase transition, induced by magnetic field, from an electron liquid to a crystalline structure has also been reported for a $2 \mathrm{D}$ electron plasma at a GaAs/AlGaAs heterojunction [7].

In finite mesoscopic systems, with small number of particles, it is, however, difficult to expect a phase transition. In these systems one observes crossovers rather than phase transitions. Therefore, the question of how the Wigner crystallization may settle down in these systems is still an intriguing fundamental problem. Leaving aside proper quantum mechanical descriptions, which due to symmetry do not allow for particle localization (see, e.g., a discussion in Ref. [8]) even a classical picture needs further clarifications. One needs to understand how a symmetry of a restricted geometry affects physical and chemical properties as a function of the number of interacting charged particles. Evidently, the decrease of system size places primary emphasis upon system boundaries. It appears that, in contrast to the $3 \mathrm{D}$ case, a $2 \mathrm{D}$ system turns out to be more complicated for studies of shell structure and the onset of crystallization in systems with charged particles of one species (see, e.g., a discussion in Ref. 9]). It is appropriate at this point to recall that, according to the Earnshaw's theorem [10, classical charges, confined in 2D hard wall, with logarithmic interparticle interaction would end up at the border of the potential (see also a discussion in Ref.[11).

Meanwhile, the question of how charged particles arrange themselves in a restricted planar geometry attracted a continuous attention for many decades (for a review see [12]). J.J. Thomson was the first to suggest an instructive solution for interacting electrons, reducing the 3D harmonic oscillator confinement to a circular (2D) harmonic oscilator [13. He developed an analytical approach which enables to trace a self-organization for a small number of electrons $(n<50)$ in a family of rings 
(shells) with a certain number of electrons in each shell (see details in 14]). Although the number of particles in outer and inner rings changes as a function of the total number of electrons, each shell is characterised by a certain discrete symmetry. In other words, $n$ point charges, located on the ring, create equidistant nodes on this ring with the angular step $\alpha=2 \pi / n$. Similar shell patterns have been found much later by means of Monte-Carlo (MC) calculations [15, 16] for charged particles (ions and electrons) confined by a 2D parabolic and hard-wall potentials (see, e.g., Refs. 17, 18 for a systematic analysis of small number of charges $n \leq 52$ ). Ground states of a few electrons in various polygons have also been analysed by means of unrestricted Hartree-Fock and densityfunctional theory calculations (e.g., 19, 20 ). Structures of polygonal patterns, similar to those obtained with an effective harmonic oscillator confinement, have been observed in experimental measurements [21, 22. In many cases, the polygonal pattern of equally charged particles is sufficiently regular.

From the above analysis, based on MC and MD calculations 23] for a relatively small number of charged particles, it follows that the number of stable configurations grows very rapidly with the number of particles. There are many local minima that have energies very close to the global minimum. These metastable states with lower (or higher) symmetry are found with much higher probabilities than the true ground state [24, 25]. This picture is akin to a liquid-solid transition, when a rapid cooling gives rise to a glasslike disordered solid rather than a crystal with lower energy. In this case various simulations techniques are too labour-intensive to be chosen for a thorough analysis of the system with increasing particle number. Evidently, a search procedure for the ground state of such systems becomes of paramount importance. One of the major aims of our paper is to provide an effective semi-analytical approach that enables us to describe ground state properties of charged particles in a circular potential as a function of particle number with a good accuracy. In order to avoid a large admixture of metastable states with the ground state we consider particles interacting by means of the Coulomb forces at zero temperature. Although we consider classical systems, our approach could shed light on the nature of self-organization of colloidal particles in organic solvents, charged nanoparticles absorbed at oil-water interfaces, electrons trapped on the surface of liquid helium or ionized plasmas. It is pertinent to note, however, that for such systems the interaction could be more complex than the one considered in our paper (cf. [26]).

For completeness we mention that similar problems have been studied in a continuous limit $27+29$. In Ref. 27 a classical hydrodynamic approach has been developed to analyze magnetoplasmonic excitations in electron quantum dots. This approach can be viewed as the simplest density-functional theory of a confined electron gas with electronic interactions treated in the Hartree approximation. A general trend of the density distribution in disk and parabolic potentials was considered in the framework of elasticity theory [28, 29]. These approaches are, however, unable to provide a detailed description of the shell structure for finite number of charged particles $(n \leq 1000)$. An asymptotic description of Coulomb systems confined by radially symmetric potentials in two and three dimensions is discussed in Ref. 30 . Although this approach is akin to ours, it lacks the detailed analysis provided in the present paper.

In order to check the validity of our theoretical approach, we also develop and perform MD calculations and compare our predictions with the MD results. In Ref.31 the reader can find our results corresponding to MD and the semi-analytical approach for $n \leq 200$ particles with a parabolic confinement.

The structure of the paper is as follows. In Sec. II we recapitulate the basic ideas of our approach, briefly discussed in Ref. 32 for disk geometry, and obtain the analytical formula for the ring-ring interaction. Sec. III is devoted to the extension of our approach for the circular parabolic potential and a comparison of the results obtained under disk and parabolic confinements. In Sec. IV we discuss the basic ideas of our MD approach and compare the results with those obtained within our semianalytical approach. The main results of our analysis are summarized in Sec. V. In two Appendixes we provide technical details and prove some statements that have been taken for granted in Ref. 32 .

\section{COULOMB INTERACTION AND CYCLIC SYMMETRY}

\section{A. Model system}

We study a system of $n$ identical charged particles with Coulomb interactions in a $2 \mathrm{D}$ confining potential. The Hamiltonian reads

$$
H=\sum_{i=1}^{n} V\left(r_{i}\right)+\alpha \sum_{i<j}^{n} \frac{1}{\left|\vec{r}_{i}-\vec{r}_{j}\right|},
$$

where $r_{i}=\left|r_{i}\right|$ is the particle distance to the center of the confining potential, and $\alpha=e^{2} / 4 \pi \varepsilon_{0} \varepsilon_{r}$ characterizes the interaction strength in the host material. Although we choose electrons as an example, the charged particles could be ions as well.

We consider two confining potentials:

1) a hard-wall (disk) confinement

$$
V(r)=\left\{\begin{array}{ll}
0, & r<R \\
\infty, & r \geq R
\end{array},\right.
$$

2) a circular parabolic potential

$$
V(r)=\frac{1}{2} m \omega_{0}^{2} r^{2} .
$$

As discussed above, in many numerical simulations, the interaction between a finite number of charged particles leads to the formation of shells in parabolic and 
disk potentials. These shells consist in a family of rings at different radii, $r_{i}$, which are occupied by a specific number of particles. In each shell $n_{i}$ point charges create equidistant nodes on the ring, with an angular spacing $\alpha_{i}=2 \pi / n_{i}$. Although a similar pattern is obtained for the parabolic and disk potentials, the distribution of particles over rings is very different in two cases. Below we will attempt to shed light on the similarity and difference in the self-organization of charged particles in both systems with aid of the semi-analytical approach. The key ingredient of that approach is an effective method for evaluating the various ring-ring energies. The method can be applied to any interaction characterized by the cyclic symmetry.

\section{B. Interaction between two rings}

We recall that the Coulomb energy of $n$ unit charges $e$, equally distributed over a ring of radius $r$, has the following form [13]:

$$
\begin{aligned}
& E_{n}(r)=\frac{\alpha}{2 r} \sum_{i=1}^{n-1} \sum_{j=i+1}^{n} \frac{1}{\sin \frac{\pi}{n}(|i-j|)}=\frac{\alpha n S_{n}}{4 r}, \\
& S_{n}=\sum_{k=1}^{n-1} \frac{1}{\sin \frac{\pi}{n} k} .
\end{aligned}
$$

Below, for the sake of discussion, we use $\alpha=1$, unless stated otherwise. For increasing number of particles several rings build up (e.g., Refs. [15,18, 23, 33]). To compute the total energy we need to add the contribution that is due to ring-ring interactions, which is absent in the Thomson model. This is the first basic ingredient of our approach.

The interaction between two rings with $n$ and $m$ point charges can be expressed as

$$
\begin{aligned}
E_{n m}\left(r_{1}, r_{2}, \psi\right) & =\sum_{i=1}^{n} \sum_{j=1}^{m} \epsilon\left(r_{1}, r_{2}, \psi_{i j}^{n m}+\psi\right) \\
& =\mathrm{G} \times \sum_{k=1}^{\mathrm{L}} \epsilon\left(r_{1}, r_{2}, \psi_{k}+\psi\right), \\
\epsilon\left(r_{1}, r_{2}, \theta\right) & =\left(r_{1}^{2}+r_{2}^{2}-2 r_{1} r_{2} \cos \theta\right)^{-1 / 2},
\end{aligned}
$$

where $\psi_{i j}^{n m}=2 \pi(i / n-j / m)$ and $\psi$ stands for the relative angular offset between both rings. Here, $\left\{\psi_{k}=\Delta \times\right.$ $k, k=1, \ldots, L\}$ and $L \equiv \operatorname{LCM}(n, m), G \equiv \operatorname{GCD}(n, m)=$ $n \times m / L$ are the least common multiple and greatest common divisor of the numbers $(n, m)$, respectively. The ring-ring energy is a periodic function with a $\Delta=2 \pi / L$ periodicity. In turn, this result shows that these kind of functions are invariant under angle transformations corresponding to the cyclic group of $L$ elements. The proof of this result is given in Appendix $\mathrm{A}$.

In virtue of the fact that the ring-ring interaction is an even periodic function in the angle $\psi$, it can be expressed by means of a cosine Fourier series

$$
E_{n m}\left(r_{1}, r_{2}, \psi\right)=\left\langle E_{n m}\right\rangle+\sum_{\ell=1}^{\infty} C_{\ell n m}\left(r_{1}, r_{2}\right) \cos (\ell L \psi) .
$$

The average value is obtained by integrating in $\psi$, and, using Eq. (6), we have

$$
\begin{gathered}
\left\langle E_{n m}\right\rangle=\frac{1}{2 \pi} \int_{0}^{2 \pi} d \psi E_{n m}\left(r_{1}, r_{2}, \psi\right) \\
=\frac{G}{2 \pi} \sum_{k=1}^{L} \int_{0}^{2 \pi} d \psi \epsilon\left(r_{1}, r_{2}, \psi_{k}+\psi\right) .
\end{gathered}
$$

All terms in the sum Eq. (9) give the same contribution, and we obtain in terms of the complete elliptic integral of first kind (see Ref.34, p. 590)

$$
\left\langle E_{n m}\right\rangle=\frac{2 n m}{\pi r_{>}(1+t)} K\left(4 t /(1+t)^{2}\right)=2 n m \frac{K\left(t^{2}\right)}{\pi r_{>}} .
$$

Here, we introduced notations: $r_{>}=\max \left(r_{1}, r_{2}\right), r_{<}=$ $\min \left(r_{1}, r_{2}\right), t=r_{</} / r_{>}$; and used the symmetry property $K\left(4 t /(1+t)^{2}\right)=(1+t) K\left(t^{2}\right)$. It is noteworthy that the average value $\left\langle E_{n m}\right\rangle$ is exactly the interaction energy between homogeneously distributed $n$ and $m$ charges over the rings.

In a similar way, the Fourier coefficients corresponding to the fluctuating part of the energy, $\Delta E_{n m}=$ $\sum_{\ell=1}^{\infty} C_{\ell n m}\left(r_{1}, r_{2}\right) \cos (\ell L \psi)$, are given by

$$
\begin{gathered}
C_{\ell n m}\left(r_{1}, r_{2}\right)=\frac{1}{\pi} \int_{0}^{2 \pi} d \psi \cos (\ell L \psi) E_{n m}\left(r_{1}, r_{2}, \psi\right) \\
=\frac{n m}{\pi} \int_{0}^{2 \pi} d \psi \frac{\cos (\ell L \psi)}{\left[r_{1}^{2}+r_{2}^{2}-2 r_{1} r_{2} \cos \psi\right]^{1 / 2}} .
\end{gathered}
$$

We have derived the following analytical expression for this integral $(\ell \geq 1$; see details in Appendix B

$$
\begin{aligned}
C_{\ell n m}\left(r_{1}, r_{2}\right)= & \frac{2 n m}{r_{>}} \frac{(2 \ell L-1) ! !}{(\ell L) !}\left(\frac{t}{2}\right)^{\ell L} \times \\
& \times{ }_{2} F_{1}\left(1 / 2, \ell L+1 / 2 ; \ell L+1 ; t^{2}\right)
\end{aligned}
$$

which is quite convenient for evaluation with symbolic algebra packages.

In particular, at $\ell=0$ one obtains the result 10 :

$$
\begin{aligned}
& { }_{2} F_{1}\left(1 / 2,1 / 2 ; 1 ; z^{2}\right)=\frac{2}{\pi} K\left(z^{2}\right) \\
& \Rightarrow C_{0 n m}\left(r_{1}, r_{2}\right)=2 n m \frac{K\left(t^{2}\right)}{\pi r_{>}} .
\end{aligned}
$$

\section{GROUND STATE CONFIGURATIONS: SEMI-ANALYTICAL APPROACH}

Before we tackle the problem of self-organization of charged particles confined in the parabolic potential, it is useful to review briefly the results obtained for the hard-wall (disk) potential. 


\section{A. Hard-wall confinement}

In this case, the total energy is defined as

$$
E_{\text {tot }}(\mathbf{n}, \mathbf{r}, \boldsymbol{\varphi})=\sum_{i=1}^{p} E_{n_{i}}+\sum_{i=2}^{p} \sum_{j=1}^{i-1} E_{n_{i} n_{j}}\left(r_{i}, r_{j}, \varphi_{i j}\right)
$$

Here, $\mathbf{n}=\left(n_{1}, \ldots, n_{p}\right)$ is a partition of the total number $n$ on $p$ rings with radii $\mathbf{r}=\left(r_{1}, \ldots, r_{p}\right)$ and offset angles between different rings $(i<j=2,3, \ldots, p)$ : $\varphi=\left(\varphi_{12}, \ldots, \varphi_{1 p}, \varphi_{23}, \ldots\right)$. We assume $R=r_{1}=1>$ $r_{2}>\cdots>r_{p}$. The numerical analysis [32] demonstrates that

$$
E_{n_{i}, n_{j}}\left(r_{i}, r_{j}, \varphi_{i j}\right) \simeq\left\langle E_{n_{i}, n_{j}}\left(r_{i}, r_{j}\right)\right\rangle
$$

holds for $n \leq 2000$ with a high accuracy. Therefore, we neglect the dependence on the relative angles $\varphi_{i j}$, i.e., the fluctuating term $\Delta E_{n_{i}, n_{j}}$. The total energy of $n$ charged particles in a disk of radius $R$ is then $E_{\text {tot }}(\mathbf{n}, \mathbf{r}, \boldsymbol{\varphi}) \simeq \mathcal{E}_{\text {avg }}(\mathbf{n}, \mathbf{r})$ with

$$
\mathcal{E}_{\text {avg }}(\mathbf{n}, \mathbf{r})=\sum_{i=1}^{p} n_{i} \frac{S_{n_{i}}}{4 r_{i}}+\frac{2}{\pi} \sum_{i<j}^{p} n_{i} n_{j} \frac{K\left(\left(r_{j} / r_{i}\right)^{2}\right)}{r_{i}} .
$$

The equilibrium configuration of particles can be obtained by minimizing Eq. (15) with respect to $(p, \mathbf{n}, \mathbf{r})$, i.e., finding the partition corresponding to the lowest total energy. For a given partition, the set of equations that determines the optimal radii $r_{i}$ is

$$
\mathcal{F}_{i}=\frac{\pi}{2}\left(r_{i} / n_{i}\right) \frac{d}{d r_{i}} \mathcal{E}_{\mathrm{avg}}(\mathbf{n}, \mathbf{r})=0, \quad i=2, \ldots, p
$$

where

$$
\begin{aligned}
\mathcal{F}_{i} & =r_{i}^{2} \sum_{j=i+1}^{p} \frac{n_{j} \mathrm{E}\left(\left(r_{j} / r_{i}\right)^{2}\right)}{r_{j}^{2}-r_{i}^{2}}-\frac{\pi}{8} S_{n_{i}}+ \\
& +r_{i} \sum_{j=1}^{i-1} n_{j}\left(\frac{r_{j} \mathrm{E}\left(\left(r_{i} / r_{j}\right)^{2}\right)}{r_{j}^{2}-r_{i}^{2}}-\frac{\mathrm{K}\left(\left(r_{i} / r_{j}\right)^{2}\right)}{r_{j}}\right)
\end{aligned}
$$

Here $\mathrm{K}=\mathrm{X}_{-1}\left(\mathrm{E}=\mathrm{X}_{1}\right)$ are complete elliptic integrals of first (second) kind: $\mathrm{X}_{p}(x)=\int_{0}^{\pi / 2} d t\left(1-x \sin ^{2} t\right)^{p / 2}$. A few standard iterations of Eqs. (16) suffice to reach an optimal energy value 15 for a given partition. By sweeping a grid of different partitions, one can readily find the lowest energy configuration for any fixed $n$.

\section{Structure of magic configurations}

Here, we consider "magic configurations" for $n \leq 395$ charges as an example. The minimization of energy with respect to the ring's partition numbers $\mathbf{n}$ leads to the following configurations:

$$
\begin{array}{cccccccccccc}
11: & 11 & & & & & & & & \\
29: & 6 & 23 & & & & & & & \\
55: & 5 & 13 & 37 & & & & & & \\
90: & 5 & 12 & 20 & 53 & & & & & \\
135: & 5 & 12 & 19 & 29 & 70 & & & & \\
186: & 5 & 12 & 19 & 26 & 37 & 87 & & & \\
246: & 5 & 12 & 18 & 25 & 34 & 46 & 106 & & \\
316: & 5 & 11 & 18 & 25 & 33 & 42 & 56 & 126 & \\
394: & 5 & 11 & 18 & 25 & 32 & 40 & 50 & 66 & 147
\end{array}
$$

These configurations are characterized by complete $p$ shells, e.g., $p=9$ for $n=394$. Our results provide an approximate formula for the number $p$ associated with a given particle number

$$
p_{H} \simeq[\sqrt{n} / 2] .
$$

Here we introduce the subindex " $\mathrm{H}$ " associated with a hard-wall potential.

If one electron is added to the configuration with complete $p$ shells, the centered hexagonal configurations (CHCs) start to appear, with a number of electrons $6 p, p=1,2,3 \ldots$, surrounding one particle at the centre. This tendency manifests clearly, starting from $n \geq 56$, i.e., we have

$$
\begin{array}{cccccccccccc}
12: & 1 & 11 & & & & & & & & \\
30: & 1 & 6 & 23 & & & & & & & \\
56: & \mathbf{1} & \mathbf{6} & \mathbf{1 2} & 37 & & & & & & \\
92: & \mathbf{1} & \mathbf{6} & \mathbf{1 2} & 20 & 53 & & & & & \\
136: & \mathbf{1} & \mathbf{6} & \mathbf{1 2} & 19 & 28 & 70 & & & & \\
187: & \mathbf{1} & \mathbf{6} & \mathbf{1 2} & \mathbf{1 8} & 26 & 37 & 87 & & & \\
248: & \mathbf{1} & \mathbf{6} & \mathbf{1 2} & \mathbf{1 8} & 25 & 34 & 46 & 106 & & \\
317: & \mathbf{1} & \mathbf{6} & \mathbf{1 2} & \mathbf{1 8} & 25 & 32 & 42 & 55 & 126 & \\
395: & \mathbf{1} & \mathbf{6} & \mathbf{1 2} & \mathbf{1 8} & \mathbf{2 4} & 32 & 40 & 50 & 65 & 147,
\end{array}
$$

with the formation of new shells and a sequence of particles in the $\mathrm{CHC}$ which is a characteristic property of the centered hexagonal lattice (CHL). Note that for $n=$ $92(1,6,12,20,53), \quad n=248(1,6,12,18,25,34,46,106)$, the onset of the $6 p$ rule needs one more particle. After formation with each new shell, these recurrent internal CHCs persist till the addition of more particles results in a sequentially increasing occupation of the inner ring, $n_{1}=2,3,4,(5)$, and back again. As expected, the span of $n$ values which exhibit this internal $\mathrm{CHC}$ increases with $p$.

This fact can be understood by considering the arrangement of the $\mathrm{CHC}$ points, $\vec{x}_{k, \ell}=k \vec{a}_{1}+\ell \vec{a}_{2}$, given by integers $k, \ell$ and the two primitive Bravais lattice vectors $\vec{a}_{1}=a(1,0)$ and $\vec{a}_{2}=a(1 / 2, \sqrt{3} / 2)$, where $a$ is the lattice constant. The $n_{p}=6 p$ sites in the $p-$ th shell are organised in different circular rings with radii $R_{k \ell}=a \sqrt{k^{2}+\ell^{2}+k \ell}$, where $p=k+\ell$ and $0 \leq \ell \leq k$, containing either 6 (if $\ell=0, k$ ) or 12 (otherwise) particles (see Fig 1 1 ). Up to $p=7$ all these radii are well ordered within and between successive shells, and the model we presented groups them in a single circular shell 

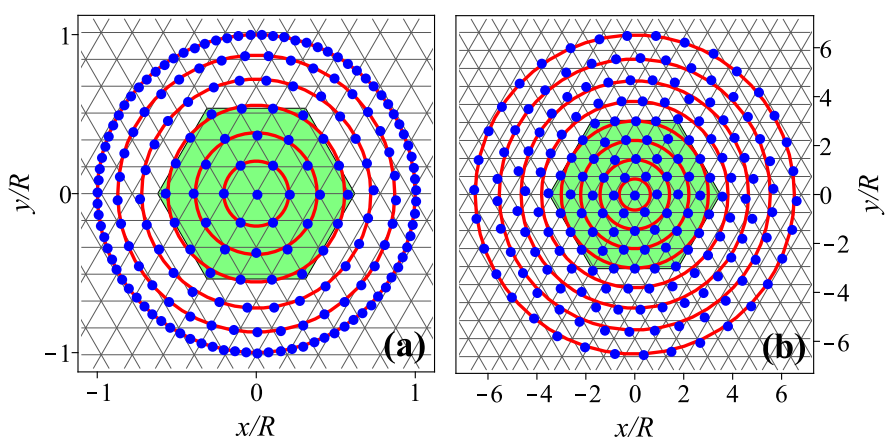

FIG. 1: (Color online) Structure of equilibrium configurations for the disk geometry (a) and for the circular parabolic potential (b) for $n=187$. In both systems there are internal (core) rings corresponding to the CHL (green region). Each shell in the core contains a family of circles with radii $R_{k \ell}$ and particle numbers $n_{\text {ring }}=6 p$ (see text). The numerical solution of Eqs. 16), 24) (rings) are compared with the MD results (dots). The calculated energies $\mathcal{E}_{\text {avg }}=23652.9 / 6099.5$ for disk/parabolic potentials are in a remarkable agreement with the corresponding MD results $\mathcal{E}_{\mathrm{MD}}=23652.2 / 6098.8$. The core region with $\{\mathbf{1}, \mathbf{6}, \mathbf{1 2}, \mathbf{1 8},(\mathbf{2 4})\}$ particles, exhibits a hexagonal pattern. The valence shells contain 87,37,26 and $34,34,34,24$ particles with an almost perfect circular structure for the disk geometry and the circular parabolic potential, respectively.

$n_{\text {ring }}=6 p$. Beyond the seventh shell, however, rings start to overlap (e.g., $R_{7,0}>R_{4,4}$ ), ultimately distorting this sequence as they depart from the centre. In other words, the $\mathrm{CHC}$ becomes broken giving up the reflection symmetry. The comparison of the equilibrium energies and configurations, calculated by means of our semianalytical model and the MD, can be found in Ref.31, We return to this point in Sec.IVB.

The systematic manifestation of the CHL with the increase of particle number $n \geq 187$ can be interpreted as the onset of the centered hexagonal crystallization in the disk geometry. We recall that for infinite systems the hexagonal lattice has the lowest energy of all twodimensional Wigner Bravais crystals 35. However, in our finite system a crossover takes place from a centered hexagonal lattice to ring localization at large $n$ with the approaching to disk boundary.

Thus, we have found a cyclic self-organization for finite number of charged particles confined in a disk geometry (cf. Refs.18, 29). For centered configurations particles localize in shells, where each $p$-th internal shell consists in particles distributed over a regular hexagon which is delimited by inscribed and circumscribed rings. This $\mathrm{CHC}$ pattern is replaced by a ring organization when approaching the boundary. A natural question arises: how general is this type of organization? Does one find a similar selforganization for other types of circular confinement? As a next example, we consider charged particles confined by an external parabolic potential.

\section{B. Parabolic potential}

In the case of a circular parabolic potential (3) the Hamiltonian (1) obeys a scaling law. We can express the energy and the coordinates in the following units

$$
R=\left(\frac{\alpha}{\beta}\right)^{1 / 3}, \quad e_{0}=\alpha^{2 / 3} \beta^{1 / 3}, \quad \beta=m \omega_{0}^{2},
$$

where $\alpha=e^{2} / 4 \pi \varepsilon_{0} \varepsilon_{r}$. In such units the Hamitonian (1) can be written in the following form

$$
\mathcal{H}=\frac{H}{e_{0}}=\frac{1}{2} \sum_{i=1}^{n} x_{i}^{2}+\sum_{i<j}^{n} \frac{1}{\left|\vec{x}_{i}-\vec{x}_{j}\right|},
$$

where $\vec{x}_{i}=\vec{r}_{i} / R$. In the form (22) the Hamiltonian does not depend on a particular value of the confinement frequency $\omega_{0}$ or the interaction strength $\alpha>0$. Therefore, the following analysis describes universal properties for this confining potential.

\section{Structure of magic configurations}

To compare the results of our model with those already obtained in the disk geometry, we also neglect the energy fluctuating term in Eq. (8). Here, we consider the ground-state configurations for $n \leq 200$ (see details in Ref.31). In contrast to the disk geometry, we are forced to find the maximal radius $r_{1}$ as a function of the particle number. In the scaled variables, within our approximation, we have for the total energy

$$
\mathcal{E}_{\text {avg }}^{S}=\mathcal{E}_{\text {avg }}(\mathbf{n}, \mathbf{x})+\frac{1}{2} \sum_{i=1}^{p} n_{i} x_{i}{ }^{2} .
$$

Here, the function $\mathcal{E}_{\text {avg }}(\mathbf{n}, \mathbf{x})$ is defined by Eq. (15), where $r_{i}$ is replaced by $x_{i}=r_{i} / R, x_{i}>x_{i+1}$. We also introduce the index "S" associated with the parabolic (soft) potential. The minimal energy configurations are obtained from the solution of the system of equations

$$
\mathcal{F}_{i}^{S}=\mathcal{F}_{i}+\frac{\pi}{2} x_{i}^{2}=0, \quad i=1, \ldots, p,
$$

where $\mathcal{F}_{i}$ is determined by Eq. (17) with $r_{i}$ replaced by $x_{i}=r_{i} / R$.

The numerical results determine the "magic configurations" with complete $p$ shells. The structures of these configurations are different from those of the hard-wall potential:

$$
\begin{array}{cccccccccc}
5: & 5 & & & & & & & \\
15: & 5 & 10 & & & & & & \\
32: & 5 & 11 & 16 & & & & & \\
52: & 5 & 11 & 16 & 20 & & & & \\
79: & 5 & 11 & 17 & 22 & 24 & & & \\
111: & 5 & 11 & 17 & 22 & 27 & 29 & & \\
148: & 5 & 11 & 17 & 22 & 28 & 32 & 33 & \\
190: & 5 & 11 & 17 & 23 & 28 & 33 & 36 & 37
\end{array}
$$


This difference manifests also in the number of rings for the same total number of particles, $n$. Our results yield the following approximate formula for the number of shells

$$
p_{S} \simeq[\sqrt{n / 2}-1] .
$$

Compared to the hard-wall potential, there are more rings in the parabolic confinement (see also Fig.11). Nevertheless, similar to the disk potential, the addition of one electron starts developing the $\mathrm{CHC}$, with internal shell occupations $6 p, p=1,2,3 \ldots$ In contrast to the hard-wall case, the onset of this $6 p$ rule is slightly delayed after the beginning of each new shell. This is reflected in the following results

$$
\begin{array}{cccccccccc}
6: & 1 & 5 & & & & & & & \\
17: & \mathbf{1} & \mathbf{6} & 10 & & & & & & \\
35: & \mathbf{1} & \mathbf{6} & \mathbf{1 2} & 16 & & & & \\
56: & \mathbf{1} & \mathbf{6} & \mathbf{1 2} & 17 & 20 & & & \\
84: & \mathbf{1} & \mathbf{6} & \mathbf{1 2} & \mathbf{1 8} & 22 & 25 & & \\
116: & \mathbf{1} & \mathbf{6} & \mathbf{1 2} & \mathbf{1 8} & 23 & 27 & 29 & & \\
155: & \mathbf{1} & \mathbf{6} & \mathbf{1 2} & \mathbf{1 8} & \mathbf{2 4} & 28 & 32 & 34 & \\
198: & \mathbf{1} & \mathbf{6} & \mathbf{1 2} & \mathbf{1 8} & \mathbf{2 4} & 29 & 33 & 37 & 38
\end{array}
$$

Note that, due to the increase in energy caused by the parabolic potential, less particles are located in this case on the rings close to the boundary. In fact the corresponding scaling for the outer shell occupations are

$$
\begin{aligned}
n_{1}^{H} & =\left[2.795 n^{2 / 3}-3.184\right], \\
n_{1}^{S} & =\left[0.2423 n^{2 / 3}+6.229 n^{1 / 3}-6.375\right] .
\end{aligned}
$$

These values are obtained from the fitting of the results at the range $n=2-400$ (300) for the hard wall (parabolic) potential. The analysis of this systematic data for the hard wall confinement also indicates that occupations for subsequent shells are quite accurately predicted $( \pm 1)$ by our model. In particular, the second shell occupation is fitted by

$$
n_{2}^{H}=\left[1.351 n^{2 / 3}-6.566\right] .
$$

In the disk geometry the energy minimization distributes a large part of particles over the perfect circular boundary. This group of charges stipulate the intrinsic ring organization in this geometry. Considering the same number of particles and system size $\left(r_{\text {ext }}^{S}=r_{\text {ext }}^{H}\right)$, in the parabolic confinement, obviously $E_{\text {Coul }}^{S}>E_{\text {Coul }}^{H}$ since the equilibrium configuration $(\mathbf{n}, \mathbf{r})_{H}$ is the one that minimizes Coulomb energy. Moreover, as a consequence of the virial theorem $\left(E_{\text {Coul }}^{S}=2 E_{\mathrm{HO}}\right)$, the total energy $E^{S}=3 E_{\text {Coul }}^{S} / 2$ is also bigger. In order to distribute the larger amount of energy in this case the system requires additional shells, absent in the disk geometry, to equilibrate the configuration. As a consequence, in the parabolic confinement the distribution of particles over rings is less inhomogeneous as compared to the disk geometry (see also Fig. 1). In turn, this feature favours the formation of a more extended CHL.

In general, the increase of particle number in a new shell disintegrates slowly the CHL in both systems. As soon as a particle appears at the center, it gives rise to the CHL again. Below, for the sake of discussion we name our semi-analytical approach as the circular model (CM).

\section{MOLECULAR DYNAMICS}

\section{A. Basic approach}

To test our results, we consider in the following both harmonic $V_{\text {ext }}(r)=1 / 2 m \omega_{0}^{2} r^{2}$ and hard-wall $V_{\text {ext }}(r)=$ $\infty \Theta(r-R)$ confinements. Finding the absolute energy minimum, $E_{\mathrm{gs}}$ of the Hamiltonian (1) is a non trivial task. The density of stable states near $E_{\mathrm{gs}}$ grows exponentially with the number of charges. Several global optimization techniques have been extensively used to this aim. Metropolis simulated annealing, with temperature $T$ as a control parameter, is particularly effective for short range forces. This method works on the basis of acceptance/rejection of a proposed change in the particle positions (and corresponding change in energy $\Delta E$ ) with probability $p(\Delta E)=\exp \left(-\Delta E / k_{\mathrm{B}} T\right)$. Random small changes over a single particle at a time are needed in order to guarantee a reasonable acceptance ratio, $r$. Usual practice adjusts the maximum change at any given $T$ as to have $r \sim 1 / 2$.

In our pursuit of the exact ground state configurations we have used a different approach, based on a quenched molecular dynamics algorithm. The method evolves the particle positions by integrating the equations of motion for the Hamiltonian (1) and adding a friction term which provides a controllable and smooth quenching of velocities

$$
m \vec{r}_{i}^{\prime \prime}=-\nabla_{i} V\left(\vec{r}_{i}\right)-b_{\mathrm{f}} \vec{r}_{i}^{\prime} .
$$

Here $V\left(\vec{r}_{i}\right)=V_{\text {ext }}\left(r_{i}\right)+\alpha \sum_{j \neq i} 1 / r_{i j}$ includes the external potential plus the Coulomb terms, and $b_{\mathrm{f}}$ is the parameter controlling the quenching of velocities. Below we discuss in detail our MD approach, using the circular parabolic potential as an example. A few results of the MD calculations for disk are presented in Refs. 25, 31, 32,

With the aid of the units 21), writing $\vec{r}=R \vec{x}, E=$ $e_{0} \mathcal{E}_{\mathrm{MD}}$ and $t=\tau / \omega_{0}$, the dynamical equations become independent of the scaling parameters for the harmonic potential

$$
\ddot{\vec{x}}_{i}=-\vec{x}_{i}+\sum_{j \neq i}^{N} \frac{\vec{x}_{i}-\vec{x}_{j}}{\left|\vec{x}_{i}-\vec{x}_{j}\right|^{3}}-\nu_{\mathrm{f}} \dot{\vec{x}}_{i},
$$

where $\dot{f} \equiv d f / d \tau$ and $\nu_{\mathrm{f}}$ is the scaled friction parameter. Given initial positions, the system (31) is evolved by standard centered 3-point derivative formulas, until 
forces over each particle are within a tolerance value (typically $\left|\ddot{\vec{x}}_{i}\right|<10^{-6}$ ). An advantage of this method over the Metropolis algorithm is that it produces a sensible global move at each iteration, thus requiring less simulation time. The performance of this method for obtaining ground state configurations is better than with usual Monte Carlo simulations, provided a sufficient number of initial conditions are tried. In fact, one of the common errors when using any of these algorithms is to be short in the number of trials and getting as a result an excited state configuration. To avoid as much as possible this scenario, we have included a systematic search of the $n$ particle configuration based on the lowest energy results for $n-1$ particles. For each of these configurations, the new particle is placed at different random positions. The new distinct configurations for the $n$ system are stored and ordered in energy to be used as starting points for the $n+1$ system. This strategy is suitable for a systematic search of ground state configurations in an ascending chain of $n$ charge particles.

\section{B. Search strategy for a single case}

In the case of a single (large) $n$ confined system, the lowest lying energy results of the circular model, with much less degrees of freedom, provide a convenient way to feed the MD analysis with sensible initial guesses. To prove this point, we have computed the probability distribution of the energy states for $n=317$ charges confined in the disk geometry with three different types of initialization. In all cases the outer shell occupation has been fixed to $n_{1}^{H}=126$ predicted by Eq. (28). In fact, this value corresponds to the actual value associated with the MD absolute energy minimum.

As it is demonstrated in Ref.25, there is a remarkable agreement between the $\mathrm{CM}$ and $\mathrm{MD}$ occupations even for a charge number of subsequent shells. Hence, we aim to assess the effectiveness of the CM prediction for $n_{2}^{H}$, Eq. 299, as a guide to initialize the external particle positions in the MD. To this end we have considered initial configurations characterized by external occupations: $n_{1}=126$ (Set 1$) ; n_{1}=126, n_{2}=55$ (Set 2); $n_{1}=126, n_{2}=56$ (Set 3 ). We have generated 3650, 2000 and 2000 configurations, respectively. In each case $n_{1}$ particles were initially set on the boundary at $r_{1}=1$, and for the last two sets $n_{2}$ particles have been homogeneously distributed at $r_{2}=0.96$.

In order to preserve these external shell occupations the $n_{1},\left(n_{2}\right)$, particles are frozen at a first stage, until the inner particles slow down (typically after some 500 time steps). At a second stage, all particles are taken into account and evolved according to Eq. (31). It is worth noticing that the chosen value $r_{2}=0.96$ is higher than the $\mathrm{CM}$ result $\left(r_{2} \simeq 0.91\right)$. The reason is twofold: i) it guarantees the desired $n_{2}$ value for the equilibrated final configuration; ii) at the beginning of the second stage it provides additional excitation energy in the form of monopole oscillations that help to access low energy states. The remaining particles are initialized randomly in the central region.

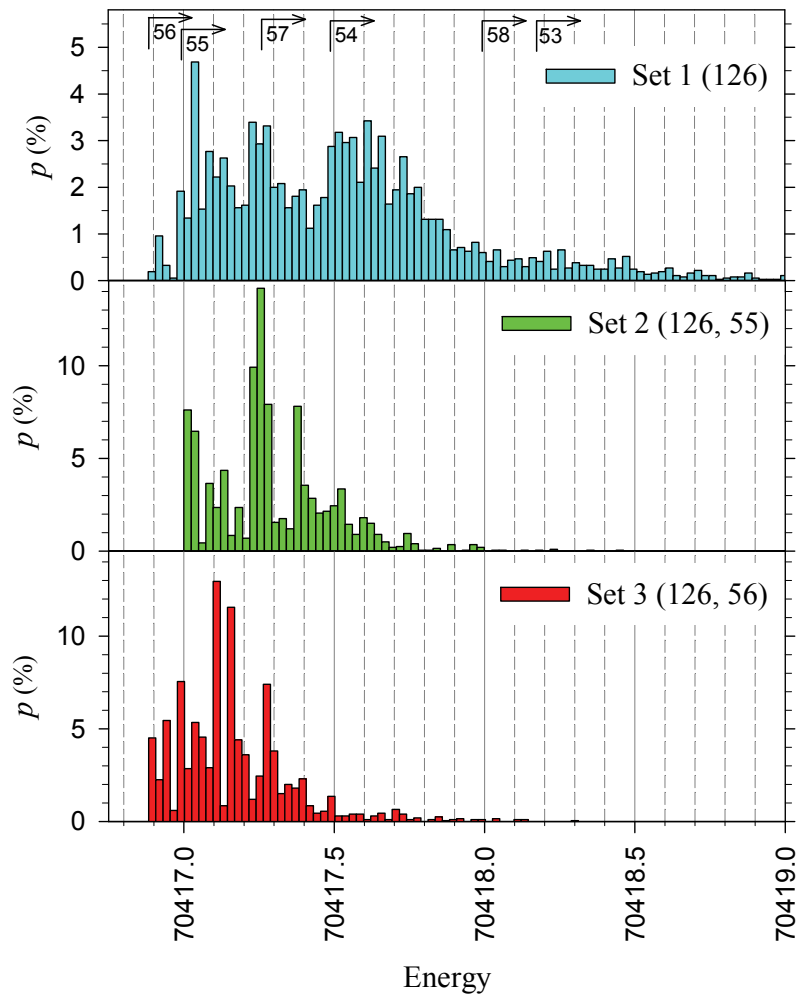

FIG. 2: (Color online) Histograms for energy states of $n=$ 317 charges confined in the disk geometry. The results are obtained with the aid of the MD method by using different initialization procedures. See text for details.

The results for the Set 1 (see Fig.2, top panel) consists of different final configurations with $n_{2}=53(5 \%), 54(36 \%), 55(38 \%), 56(18 \%), 57(3 \%)$ and $58(0.2 \%)$. The arrows indicate the starting energy for each $n_{2}$ value. In this case the low energy states are dominated by configurations with $n_{2}=(55,56)$, although just about one out of five runs leads to the $n_{2}$ value shared by the ground state at $\mathcal{E}_{\mathrm{MD}}=70416.883$. The realization of the ground state does not exceed $0.2 \%$. The Set 2 (Fig.2 middle panel) provides the lowest state $\mathcal{E}_{\mathrm{MD}}=70417.000$ with $n_{2}=55$ (the $\mathrm{CM}$ partition), which is slightly above the true ground state. The Set 3 (Fig. 2, bottom panel) explores the nearby $n_{2}=56$ configurations, with $n_{2}=n_{2}^{H}$ provided by Eq. (29). In this case the absolute energy minimum is found with a probability $\sim 4.5 \%$ which is higher by a factor 25 relative to the probability found for the Set 1 . Thus, even if one has to check nearby $n_{2}^{H} \pm 1$ values, the scanning effort clearly benefits from the scalings found within the CM.

For the sake of illustration, we present in Fig. 3 the distribution of charges in the equilibrium configuration obtained by means of our procedure for $n=317$. Three 
regions are found (see Fig. 3 p). The central region (green colored hexagonal area) is comprised of the almost perfect CHL with 1,6 and 12 particles followed by a third shell containing 19 (instead of 18) particles. This additional charge (at the center of the small yellow circle) breaks the hexagonal structure. Its effect propagates to the middle region. Here, together with additional defects, it builds still a hexagonal, although deformed, structure (gray lines are used to indicate the deformed lattice). The last region contains three external rings with 126,56,42 particles. Applying a simple clustering algorithm, that will be discussed below, we can identify circular shells in the MD results (see Fig. 3 3 ). As a result, we obtain the following configuration $(126,56,42,33,22,19,12,6,1)$ compatible with the CM result $(126,55,42,32,25,18,12,6,1)$ (see also [31).

Below, we compare the $\mathrm{MD}$ and the $\mathrm{CM}$ results in more details.
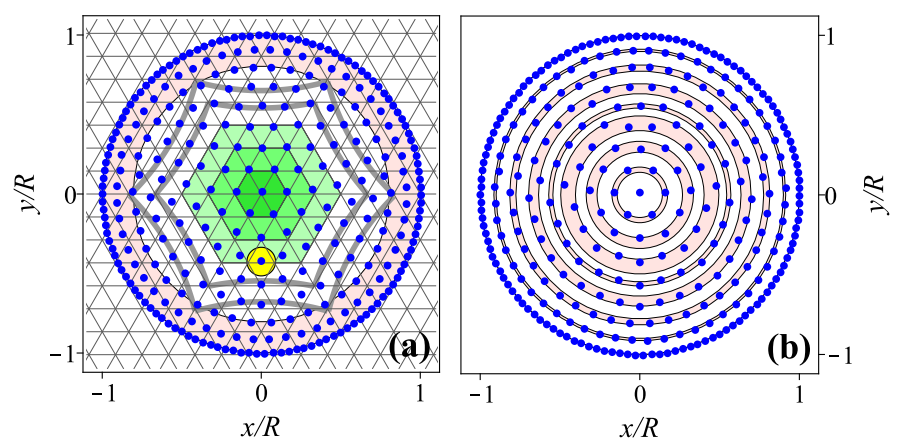

FIG. 3: (Color online) Structure of equilibrium configurations for the disk geometry obtained by means of the MD for $n=317$. (a) Distribution of particles versus hexagonal grid. The core (green) region with $\{\mathbf{1}, \mathbf{6}, \mathbf{1 2})$ particles exhibits a hexagonal pattern. The additional particle (displayed within the small yellow circle) in the third shell breaks the hexagonal $6 p$ rule. The intermediate region can still be associated with a deformed hexagonal lattice (gray lines to guide the eye). The valence shells contain $126,56,42$ charges with an almost perfect circular structure. (b) Distribution of particles on various rings, found with the clustering algorithm described in Sec. IV.C, with number of charges: $126,55,42,32,25,18,12,6,1$.

\section{Molecular dynamics and the circular model}

The numerical solution of the system (15, 17) for the hard confinement provides a remarkable agreement with the MD calculations for equilibrium configurations up to $n=105$, excluding a few cases (see Table I in Ref.32). Our MD results agree with those of Ref.23 up to $n=160$ particles, while we obtain lower energies for $n=400,500,1000$ and also systematically better values for $n>52$ than those implied in Fig. 8 of Ref.29, In Ref. 25 the reader can find the comparison of the MD and the $\mathrm{CM}$ results for the disk geometry for $161 \leq n \leq 260$.
In that paper, we have also demonstrated the usefulness of the CM to speed up the random search method of the true ground state for $n=395$ in the MD calculations, found in Ref.[24].

TABLE I: Values for the only eleven cases where optimal configurations, obtained with the aid of Eq. 24, disagree with the MD results. The systematic MD results for $n \leq 52$ can be found also in Ref. [17.

\begin{tabular}{|c|c|c|c|}
\hline$n$ & $\mathcal{E}^{S}{ }_{\text {avg }}(n)$ & $\delta$ & configuration \\
\hline 19 & 115.1127 & 0.0208 & $(11,7,1)_{2}^{1}$ \\
22 & 149.7743 & 0.0571 & $(13,8,1)_{1}^{3}$ \\
32 & 290.7905 & 0.0655 & $(16,11,5,0)_{1}^{4}$ \\
34 & 323.4146 & 0.0537 & $(16,11,6,1)_{1}^{2}$ \\
36 & 357.5053 & 0.0313 & $(16,12,7,1)_{3}^{1}$ \\
39 & 411.3570 & 0.0392 & $(18,13,7,1)_{1}^{4}$ \\
40 & 430.0216 & 0.0924 & $(18,13,8,1)_{1}^{4}$ \\
41 & 449.0085 & 0.1290 & $(18,13,8,2)_{1}^{2}$ \\
46 & 548.6758 & 0.0976 & $(18,15,9,4)_{4}^{1}$ \\
52 & 678.9715 & 0.0671 & $(20,16,11,5,0)_{1,2}^{4,5}$ \\
53 & 701.8207 & 0.1162 & $(19,16,11,6,1)_{1}^{2}$ \\
\hline
\end{tabular}

In the case of the parabolic potential we obtain good agreement with the MD results as well, excluding a few cases (see Table $I$ ) up to $n \approx 51$. The difference $\delta=$ $\mathcal{E}^{S}{ }_{\text {avg }}-\mathcal{E}_{\mathrm{MD}}$ provides the error of our approximation. The rings are counted starting from the external one which is the first ring. The notation $(11,7,1)_{2}^{1}$ means that we have to add one particle in the first ring and remove one particle from the second ring in order to obtain the MD result. Although the total energy errors are very small, the assumptions of our model fail to predict the correct configurations for shown total $n$.

Since in the disk geometry the external radius is fixed $(R=1)$, the parabolic potential has one more degree of freedom in terms of collective variables. It is natural to assume that this degree of freedom is related to fluctuations of the external ring radius around some equilibrium radius value (radial fluctuations). As a result, such a motion creates fluctuations in the particle number around some optimal value in the external ring affecting the particle number in the closest ring.

In order to get deeper insight into this phenomenon we have applied a simple clustering algorithm to identify the formation of circular shells in the MD results. At a first stage, we order particles according to their distance to the centre $r_{1} \leq r_{2} \leq \cdots \leq r_{n}$. Next, we define the gaps between consecutive particles $\delta_{i}=r_{i+1}-r_{i}$ and sort them by decreasing value, i.e., $\delta_{i_{1}} \geq \delta_{i_{2}} \geq \cdots \geq \delta_{i_{n-1}}$. By defining the function

$$
F_{\mathrm{MD}}(p)=\frac{r_{1}+\sum_{k=1}^{p-1} \delta_{i_{k}}}{p},
$$

the optimal groups are found by maximizing the average spatial separation between consecutive groups with respect to the number of shells $p$. Additionally, we impose 
the constraint $0 \leq p(n)-p(n-1) \leq 1$ and detect when a new particle settles at the centre of the structure, $r_{1} \simeq 0$, thus opening a new shell. Once the number of shells is obtained, the related function

$$
R_{\mathrm{MD}}(n)=\frac{p F_{\mathrm{MD}}(p)}{r_{n}}
$$

provides a simple measure of how close the MD particle configuration is to a well defined ring structure. Notice that for strict circular configurations, such as those provided by our circular model, $R_{\mathrm{MD}}(n)=1$. A significant departure from this maximum value would imply that the particle distribution deviates from the prediction of the CM.
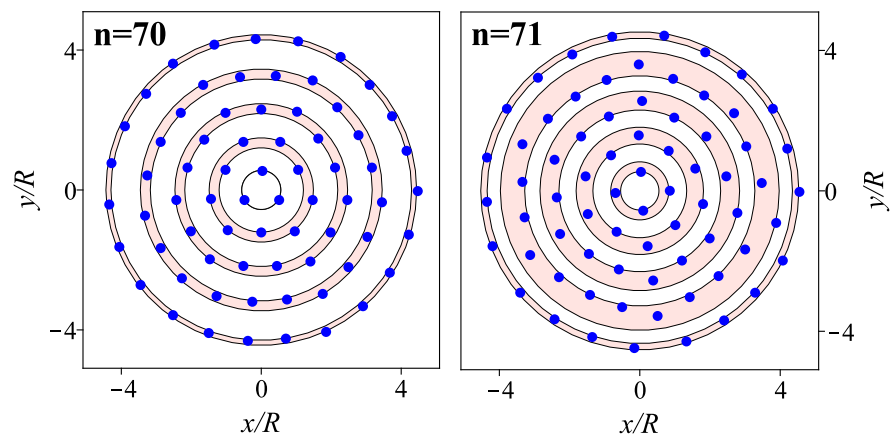

FIG. 4: (Color online) Structure of the MD equilibrium configurations for $n=70,71$ charged particles in the circular parabolic potential. In both systems five rings are identified. For $n=70$ the MD configuration is $\{\mathbf{3}, \mathbf{9}, \mathbf{1 5}, \mathbf{2 0}, \mathbf{2 3}\}$, $\mathcal{E}_{\mathrm{MD}}=1135.298$. The circular model predicts the same configuration with $\mathcal{E}_{\text {avg }}^{S}=1135.474$. For $n=71$ the MD (the circular model) configuration is $\{\mathbf{4}, \mathbf{1 0}, \mathbf{1 5}, \mathbf{2 1}, \mathbf{2 1}\}$ $(\{\mathbf{4}, \mathbf{9}, \mathbf{1 5}, \mathbf{2 0}, \mathbf{2 3}\}), \mathcal{E}_{\mathrm{MD}}\left(\mathcal{E}_{\mathrm{avg}}^{S}\right)=1163.410(1163.579)$.

This fact is illustrated in Fig. 4 where the result of this clustering algorithm is shown for $n=70,71$. The actual values $R_{\mathrm{MD}}(70)=0.775$ and $R_{\mathrm{MD}}(71)=0.520$ indicate that the system with $n=70$ is quite well described by a ring structure. It is not the case, however, for $n=71$, where the resulting shells have much larger widths. The addition of one particle produces a visible finite size effect which transforms the system from a rigid ring organization to a kind of glasslike behavior. In Fig. 5 the function (33) shows that the MD configurations form a robust circular structure for the disk geometry; its value remains close to the maximum ( $\geq 90 \%$ ) for $n \leq 100$ charged particles. The accumulation of a big fraction of particles on the perfect circular boundary strongly constrains the internal ring organization. In this case the circular model provides a remarkable agreement with the MD results both for the equilibrium configurations and their energies.

Conversely, while this function does frequently not drop down below $(\simeq 60 \%)$ in the case of the parabolic potential, there are a few cases $n=39,52,62,65,71,75,90 \ldots$

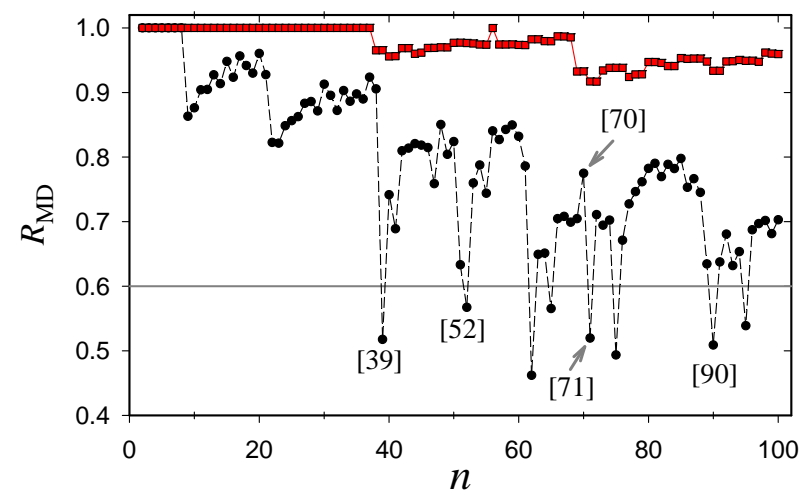

FIG. 5: (Color online) The function (33) vs number of charged particles. The results for the disk geometry and the parabolic potential are shown by (red) squares and (black) circles. The arrows indicate the location of $n=70,71$ charge particle configurations discussed on Fig 4

that exhibit a visible deviation from a circular distribution. In these cases the system manifests detectable fluctuations in particle positions, driven by a change in the central structure $\left(n_{p}=1 \rightarrow 2\right.$ or $\left.n_{p}>1 \rightarrow 1\right)$, that affect the width of the corresponding shells. In general, one observes a kind of cold melting of the system configuration that preserves, however, to some extent the ring structure.

A comparison of the MD results with those of the circular model for the total energy demonstrates a remarkable agreement. Although there is a small disagreement between the results obtained within our model and MD calculations for the ground state configurations, it is noteworthy that the onset of the centered hexagonal lattice in the MD calculations for large $n$ has been clearly recognized with the aid of the circular model.

\section{SUMMARY}

We have developed the method (see Sects. II, III) which allows us to analyze the equilibrium formation and filling of rings with a finite number of particles interacting by means of the Coulomb forces in the case of a circular lateral confinement. As an example, we analyzed disk and parabolic confinements. Our approach is based on the cyclic symmetry of the Coulomb energy between particles distributed over different rings. As a result, the problem of $n$ interacting charged particles is reduced to the description of $p(\ll n)$ rings, with homogeneously distributed integer charges. To test the validity of the method we have also developed the MD approach (Sec. IV) and present the comparison of the results in Ref.31.

We have demonstrated that our method is good enough to obtain exact ground state configurations with correct energies, excluding a few particular cases, up to $n \leq 105$ 
and $\leq 51$ for a hard and parabolic confinements, respectively. For bigger systems the solution of the model equations provide also very good approximations to the exact ground state configurations. Indeed, the energy errors do not exceed a small percentage fraction of the exact values. However, this achievement is a necessary but not a sufficient condition to conclude that the CM is effective. The systematic analysis of the CM results provides the estimate for a number of charges $n_{1}$ distributed over the external (boundary) ring for large systems $(n \leq 400)$. Once this number is identified, one has to fix the number of charges on the second ring, the nearest neighbor to the boundary one, based on the CM prediction $n_{2} \pm 1$. At the same time, the inner shell structure, as predicted by the CM, should be disregaded, since it may introduce a bias. With the aid of this strategy the computational effort to get global energy minima is much less than in the MD or simulated annealing (SA) calculations. In fact, both methods are flawed by two major problems. First, due to the long range of Coulomb forces, computing time grows with the number of particles as $n^{3}$. Second, and more important, the number of equilibrium configurations near the absolute energy minimum grows exponentially with $n$. This last fact increases considerably the computational efforts needed to avoid getting stuck at local energy minima, because many different initial condition simulations are needed. Therefore, we believe that an accurate method, capable of explaining shell structure, which works with a much reduced number of variables $(\sqrt{n / 2}$ vs $n)$ is a remarkable achievement. Moreover, the results obtained by means of our method can be used to feed SA or MD calculations with sensible initial configurations, reducing substantially the amount of scanning normally needed to visit the global energy minima.

In our circular model each $p$-th shell consists of a set of point charges distributed over a regular hexagon inscribed and circumscribed by two circles. Based on the results of our analysis, we have found that in both potentials the increase of particle number leads to the onset of a centered hexagonal lattice that transforms smoothly to a few circular rings at the boundary. A similar conclusion has been drawn on the basis of the Monte Carlo calculations for $n>150$ charges confined by a circular parabolic potential 33, although the authors admitted that their equilibrium configurations are not necessarily true ground states. Based on our results we speculate that this self-organization should be typical for any 2D finite system of identical charges confined by a circularly symmetric potential. We recall, however, that depending on the size of the system one has to take into account the onset of quantum correlations for increasing particle number at a fixed system size (see a discussion in Ref. 32).

\section{Acknowledgments}

We thank Kostya Pichugin for useful discussions, and F.M. Peeters for bringing to our attention Ref 33, M.C. is grateful for the warm hospitality at JINR. This work was supported in part by Bogoliubov-Infeld program of BLTP and Russian Foundation for Basic Research.

\section{Appendix A: Cyclic symmetry and the Coulomb sums}

We want to prove that

$\sum_{i=1}^{n} \sum_{j=1}^{m} \mathrm{~F}\left[\cos \left(2 \pi\left(\frac{i}{n}-\frac{j}{m}\right)\right)\right]=G \sum_{k=1}^{L} \mathrm{~F}\left[\cos \left(2 \pi \frac{k}{L}\right)\right]$,

where $L \equiv \operatorname{LCM}(n, m)$ and $G \equiv \operatorname{GCD}(n, m)=n \times m / L$ are the least common multiple and greatest common divisor of the of numbers $(n, m)$, respectively.

\section{Proof:}

Due to the fact that $\mathrm{F}$ is a function (every element belonging to the domain is related to a unique element of the image set) it suffices to prove that the multiset [36] of angles $A=\left\{2 \pi\left(\frac{i}{n}-\frac{j}{m}\right), i=1, \ldots, n \wedge j=1, \ldots, m\right\}$ is equal, under the cyclic symmetry, to the multiset $B=$ $\left\{2 \pi \frac{k}{L}, k=1 \ldots L\right\}$, where each element is repeated $G$ times.

In order to take into account the cyclic symmetry explicitly, we change the numbers $k$ by their associated equivalence classes, defined as $C_{k}=\{k+\gamma L, \forall \gamma \in \mathbb{Z}\}$. As a result, a $k$-value appearing inside the $B$ multiset has to be read as an unspecified member of the $C_{k}$ class. The set composed by all these classes constitutes a partition of $\mathbb{Z}$ into $L$ classes.

Since any linear combination of two integers $(n, m)$ is equal to a multiple of its greatest common divisor $G=$ $\operatorname{GCD}(n, m)$, we have

$$
\frac{i}{n}-\frac{j}{m}=\frac{i m-j n}{n m}=\frac{k G}{n m}=\frac{k G}{L G}=\frac{k}{L},
$$

where $k$ is an integer. Evidently, this result allows us to change two sums over the variables $i$ and $j$ by a single suitable sum over the variable $k$.

Note that for the multiset of angles $A$ the substitutions

$$
i \rightarrow i+\alpha n \quad \text { and } \quad j \rightarrow j+\beta m,
$$

where $\alpha, \beta \in \mathbb{Z}$, have no practical effect. Furthermore, since a pair of indices $(i, j)$ produces an index $k$ that belongs to the class $C_{k}$, the shifted pair of indices $(i+$ $\alpha n, j+\beta m)$ produces an index $k^{\prime}$ that also belongs to the class $C_{k}$ :

$$
\begin{gathered}
(i+\alpha n) m-(j+\beta m) n=i m-j n+(\alpha-\beta) L G= \\
(k+(\alpha-\beta) L) G .
\end{gathered}
$$


Evidently, there are exactly $L$ different $C_{k}$ classes.

Now we show that each class contains $G$ different elements. If there exists a pair $(i, j)$ such that

$$
i m-j n=k G,
$$

then there are exactly $G$ pairs $\left(i^{\prime}, j^{\prime}\right)$, with the restrictions $1 \leq i^{\prime} \leq n$ and $1 \leq j^{\prime} \leq m$, such that $i^{\prime} m-j^{\prime} n=$ $k G$. Note that if $(i, j)$ satisfy $\mathrm{A} 2$, then all couples of the form

$$
\left(i^{\prime}=i+l \frac{n}{G}, j^{\prime}=j+l \frac{m}{G}\right)
$$

where $l$ is an arbitrary integer, also satisfy A2, because $i^{\prime} m-j^{\prime} n=\left(i+l \frac{n}{G}\right) m-\left(j+l \frac{m}{G}\right) n=i m-j n=k G$.

If we restrict the values of $l$ to $0 \leq l \leq G-1$, i.e. $G$ different choices, the resulting values $i^{\prime}$ and $j^{\prime}$ are all different. Indeed, in this situation, if $G>1$, the following inequalities hold

$$
\frac{n}{G} \leq\left|i^{\prime}-i^{\prime \prime}\right|<n \quad \text { and } \quad \frac{m}{G} \leq\left|j^{\prime}-j^{\prime \prime}\right|<m .
$$

If $l=G+\lambda$, with $\lambda=0,1,2, \ldots$ the indices are cyclicly equivalent

$$
i+\frac{(G+\lambda) n}{G}=i+n+\frac{\lambda n}{G} \sim i+\frac{\lambda n}{G} .
$$

Therefore, since there are $n m$ pairs, every one of the $L$ different $C_{k}$ classes must contain $G$ pairs. This completes the proof. The fact that we consider a cosine function is irrelevant, since the requirement that $\epsilon\left(r_{1}, r_{2}, \theta\right)$ to be periodic is enough.

\section{Appendix B: Coefficients of the Fourier transform}

In order to find an analytical expression for the integral (11), we employ the Legendre expansion for the Coulomb potential

$$
\frac{1}{\left[r_{1}^{2}+r_{2}^{2}-2 r_{1} r_{2} \cos \psi\right]^{1 / 2}}=\frac{1}{r_{>}} \sum_{u=0}^{\infty} t^{u} P_{u}(\cos \psi),
$$

where $r_{>}=\max \left(r_{1}, r_{2}\right), r_{<}=\min \left(r_{1}, r_{2}\right), t=r_{<} / r_{>}$. With the aid of the cosine series for the Legendre polynomials (see Ref.34, p. 776)

$$
P_{u}(\cos \psi)=\frac{1}{4^{u}} \sum_{v=0}^{u}\left(\begin{array}{c}
2 v \\
v
\end{array}\right)\left(\begin{array}{c}
2(u-v) \\
u-v
\end{array}\right) \cos ((u-2 v) \psi)
$$

we obtain for Eq. 11 the following form

$$
\begin{aligned}
C_{\ell n m}\left(r_{1}, r_{2}\right) & =\frac{n m}{r_{>} \pi} \sum_{v=0}^{\infty}\left(\frac{t}{4}\right)^{v}\left(\begin{array}{c}
2 v \\
v
\end{array}\right) \sum_{w=0}^{v}\left(\frac{t}{4}\right)^{w}\left(\begin{array}{c}
2 w \\
w
\end{array}\right) \\
& \times \int_{0}^{2 \pi} \cos [(w-v) \psi] \cos (\ell L \psi) d \psi, \quad(\mathrm{B} 1
\end{aligned}
$$

where $u=w+v$. The integral splits in two terms, since $\cos A \cos B=[\cos (A+B)+\cos (A-B)] / 2$. In order to have a nonzero value, the first term with the argument $w=v-l L$ in the cosine function requires $v \geq l L$. As a result, we have

$$
C_{\ell n m}\left(r_{1}, r_{2}\right)=\frac{n m}{r_{>}} \sum_{v=\ell L}^{\infty}\left(\frac{t}{4}\right)^{2 v-\ell L}\left(\begin{array}{c}
2 v \\
v
\end{array}\right)\left(\begin{array}{c}
2(v-\ell L) \\
v-\ell L
\end{array}\right)
$$

$$
=\frac{n m}{r_{>}}\left(\frac{t}{4}\right)^{\ell L} \sum_{k=0}^{\infty}\left(\begin{array}{c}
2(\ell L+k) \\
\ell L+k
\end{array}\right)\left(\begin{array}{c}
2 k \\
k
\end{array}\right)\left(\frac{t}{4}\right)^{2 k},
$$

where $k=v-\ell L$. The second term yields the same result, that duplicates the expression $(\mathrm{B} 2)$.

This double sum can be expressed in terms of the hypergeometric function. In virtue of the identity

$$
\frac{1}{4^{p}}=\frac{\Gamma(p+1 / 2)}{\sqrt{\pi} p !}
$$

we arrive at the final form

$$
\begin{aligned}
& C_{\ell n m}\left(r_{1}, r_{2}\right)= \\
& =\frac{2 n m}{r_{>}} \frac{t^{\ell L}}{\pi} \sum_{k=0}^{\infty} \frac{\Gamma(\ell L+k+1 / 2) \Gamma(k+1 / 2)}{\Gamma(\ell L+k+1) k !} t^{2 k} \\
& =\frac{2 n m}{r_{>}} \frac{t^{\ell L}}{\sqrt{\pi}} \frac{\Gamma(\ell L+1 / 2)}{(\ell L) !} \sum_{k=0}^{\infty} \frac{(1 / 2)_{k}(\ell L+1 / 2)_{k}}{(\ell L+1)_{k}} \frac{t^{2 k}}{k !} \\
& =\frac{2 n m}{r_{>}} \frac{(2 \ell L-1) ! !}{(\ell L) !}\left(\frac{t}{2}\right)^{\ell L} \times \\
& \quad \times{ }_{2} F_{1}\left(1 / 2, \ell L+1 / 2 ; \ell L+1 ; t^{2}\right) .
\end{aligned}
$$

Here, we have used a definition of the hypergeometric function in terms of the Pochhammer symbol $(a)_{b}=$ $\Gamma(a+b) / \Gamma(a)$.

At $\ell L \gg 1$ one can use the asymptotic value for the central binomial coefficients $\left(\begin{array}{c}2 M \\ M\end{array}\right) /\left(4^{M}\right) \approx 1 / \sqrt{\pi M}$ , where $M=\ell L$. As a result, we obtain the asymptotic limit of Eq. B3):

$$
C_{\ell n m}\left(r_{1}, r_{2}\right) \approx \frac{2 n m}{\sqrt{\pi} r_{>}} \sum_{k=0}^{\infty} \frac{\left(\begin{array}{c}
2 k \\
k
\end{array}\right)}{4^{k}} \frac{t^{\ell L+2 k}}{\sqrt{\ell L+k}}
$$

This result shows explicitly the decreasing magnitude of the coefficients accompanying the powers of the variable $t$ in Eq. 12 with the increase of the product $\ell L$. 
[1] J.L. Birman, R.G. Nazmitdinov, and V.I. Yukalov, Phys. Rep. 526, 1 (2013).

[2] H. Saarikoski, S.M. Reimann, A. Harju, and M. Manninen, Rev. Mod. Phys. 82, 2785 (2010).

[3] E. Wigner, Phys. Rev. B46, 1002 (1934).

[4] J. Cioslowsky, J. Chem. Phys. 133, 234902 (2010).

[5] H. Totsuji, T. Kishimoto, C. Totsuji, and K. Tsuruta, Phys. Rev. Lett. 88, 125002 (2002).

[6] C.C. Grimes and G. Adams, Phys. Rev. Lett. 42, 795 (1979).

[7] E.Y. Andrei, G. Deville, D.C. Glattli, F.I.B. Williams, E. Paris, and B. Etienne, Phys. Rev. Lett. 60, 2765 (1988).

[8] Ll. Serra, R. G. Nazmitdinov, and A. Puente, Phys.Rev. B 68, 035341 (2003).

[9] J. Cioslowsky and J. Albin, J. Chem. Phys. 136, 114306 (2012).

[10] S. Earnshaw, Trans. Cambridge Philos. Soc. 7, 97 (1842).

[11] Y. Levin and J.J. Arenzon, Europhys. Lett. 63, 415 (2003).

[12] M.J. Bowick and L. Giomi, Adv. Phys. 58, 449 (2009).

[13] J. J. Thomson, Phil. Mag. 7, 237 (1904).

[14] B. Partoens and F.M. Peeters, J. Phys.: Condens. Matter 9, 5383 (1997).

[15] Yu.E. Lozovik and V.A. Mandelshtam, Phys. Lett. A 165, 469 (1992).

[16] F. Bolton and U. Rössler, Superlatt. Microstruct. 13, 139 (1992).

[17] V.M. Bedanov and F.M. Peeters, Phys. Rev. B 49, 2667 (1994).

[18] M. Kong, B. Partoens, A. Matulis, and F.M. Peeters, Phys. Rev. E 69, 036412 (2004).

[19] E. Räsänen, H. Saarikoski, M.J. Puska, and R.M. Nieminen, Phys. Rev. B 67, 035326 (2003).

[20] M. Ishizuki, H. Takemiya, T. Okunishi, K. Takeda, and K. Kusakabe Phys. Rev. B 85, 155316 (2012).

[21] M. Saint Jean, C. Even, and C. Guthmann, Eur. Phys. Lett. 55, 45 (2001).

[22] E. Rousseau, D. Ponarin, L. Hristakos, O. Avenel, E. Varoquaux, and Y. Mukharsky, Phys. Rev. B 79, 045406 (2009).

[23] A. Worley, arXiv: physics/060923 (2006).

[24] P. Amore, Phys. Rev. E 95, 026601 (2017).

[25] A. Puente, R.G. Nazmitdinov, M. Cerkaski, and K.N. Pichugin, Phys. Rev. E 95, 026602 (2017).

[26] M. Girotto, A.P. dos Santos, and Y. Levin, J.Phys. Chem.B120, 5817 (2016).

[27] Z.L. Ye and E. Zaremba, Phys. Rev. B 50, 17217 (1994).

[28] A.A. Koulakov and B.I. Shklovskii, Philos. Mag. B 77, 1235 (1998); Phys. Rev. B 57, 2352 (1998).

[29] A. Mughal and M.A. Moore, Phys. Rev. E 76, 011606 (2007).

[30] J. Cioslowski and J. Albin, J. Chem. Phys. 139, 114109 (2013).

[31] See Supplemental Material for comparison of equilibrium configurations and energies obtained by means of the MD and the CM calculations.

[32] M. Cerkaski, R.G. Nazmitdinov, and A.Puente, Phys. Rev. E 91, 032312 (2015).

[33] M. Kong, B. Partoens, and F.M. Peeters, Phys. Rev. E 67, 021608 (2003).

[34] M. Abramowitz and I. A. Stegun, Handbook of Math- ematical Functions with Formulas, Graphs, and Mathematical Tables Tenth Printing (National Bureau of Standards, Applied Mathematics Series 55, U.S. Government Printing Office, Washington, D.C., 1972).

[35] L. Bonsall and A.A. Maradudin, Phys. Rev. B 15, 1959 (1977).

[36] A multiset is a set which can contain repeated elements. 


\section{Supplemental Material: The MD and Circular Model results}

\section{I: Disk geometry}

These tables summarize our results corresponding to the minimum energy equilibrium configurations under disk confinement, discussed in Sec.IIIA.

DATA for Table (Eq. (18))

\begin{tabular}{|c|c|c|c|c|}
\hline $\mathrm{n}$ & $\mathrm{CM}$ energies & Configuration & MD energies & Configuration \\
\hline 11: & 48.57568 & {$[11]$} & 48.57568 & {$[11]$} \\
\hline 29: & 444.5491 & {$[23,6]$} & 444.5478 & {$[23,6]$} \\
\hline 55: & 1792.007 & {$[37,13,5]$} & 1791.974 & {$[37,13,5]$} \\
\hline 90: & 5115.563 & {$[53,20,12,5]$} & 5115.408 & {$[52,20,12,5,1]$} \\
\hline 135: & 11995.371 & {$[70,29,19,12,5]$} & 11994.978 & {$[70,28,19,12,5,1]$} \\
\hline 186: & 23391.044 & {$[87,37,26,19,12,5]$} & 23390.284 & {$[87,37,26,18,11,6,1]$} \\
\hline 246: & 41743.132 & {$[106,46,34,25,18,12,5]$} & 41741.995 & {$[106,46,34,25,16,12,6,1]$} \\
\hline 316: & 69962.348 & {$[126,56,42,32,25,18,11,5]$} & 69960.435 & {$[126,55,42,33,26,15,12,6,1]$} \\
\hline 394: & 110093.60 & {$[147,66,50,40,32,25,18,11,5]$} & 110090.41 & {$[147,66,50,40,26,26,19,13,6,1]$} \\
\hline
\end{tabular}

DATA for Table (Eq. (20))

\begin{tabular}{|c|c|c|c|c|}
\hline $\mathrm{n}$ & CM energies & Configuration & MD energies & Configuration \\
\hline 12: & 59.57568 & {$[11,1]$} & 59.57568 & {$[11,1]$} \\
\hline 30: & 479.0854 & {$[23,6,1]$} & 479.0796 & {$[23,6,1]$} \\
\hline $56:$ & 1862.734 & {$[37,12,6,1]$} & 1862.650 & {$[37,12,6,1]$} \\
\hline 92: & 5358.578 & {$[53,20,12,6,1]$} & 5358.353 & {$[53,20,12,6,1]$} \\
\hline 136: & 12181.755 & {$[70,28,19,12,6,1]$} & 12181.345 & {$[70,28,19,12,6,1]$} \\
\hline 187: & 23652.947 & {$[87,37,26,18,12,6,1]$} & 23652.188 & {$[87,37,26,18,12,6,1]$} \\
\hline 248: & 42447.440 & {$[106,46,34,25,18,12,6,1]$} & 42446.278 & {$[107,46,34,25,17,12,6,1]$} \\
\hline 317: & 70418.854 & {$[126,55,42,32,25,18,12,6,1]$} & 70416.883 & {$[126,56,42,33,22,19,12,6,1]$} \\
\hline 395: & 110667.59 & {$[147,65,50,40,32,24,18,12,6,1]$} & 110664.44 & {$[147,66,51,40,26,26,19,13,6,1]$} \\
\hline
\end{tabular}




\section{II: Parabolic confinement}

These tables summarize our results corresponding to the minimum energy equilibrium configurations under parabolic confinement. $E_{\mathrm{MD}}, E_{\mathrm{CM}}$ are the total MD and the Circular Model energies. $R_{\text {avg }}$ and $R_{\text {ext }}$ are respectively the average radius for the external shell in MD and the external CM radius. All quantities are expressed in parabolic units, defined in the main text. The approximate shell configuration in MD has been obtained by the algorithm described in Sec.IVB. Notice that for specific values of $n$ (e.g., $n=90$ ) some intermediate shells appear grouped together, indicating rather big particle dispersions within those shells.

\begin{tabular}{|c|c|c|c|c|c|c|}
\hline$n$ & $E_{\mathrm{MD}}$ & Configuration & $R_{\text {avg }}$ & $E_{\mathrm{CM}}$ & Configuration & $R_{\text {ext }}$ \\
\hline 2 & 1.190551 & [2] & 0.630 & 1.190551 & [2] & 0.630 \\
\hline 3 & 3.120126 & [3] & 0.833 & 3.120126 & [3] & 0.833 \\
\hline 4 & 5.827177 & [4] & 0.985 & 5.827177 & [4] & 0.985 \\
\hline 5 & 9.280127 & [5] & 1.112 & 9.280127 & [5] & 1.112 \\
\hline 6 & 13.35587 & {$[5,1]$} & 1.334 & 13.35587 & {$[5,1]$} & 1.334 \\
\hline 7 & 17.99543 & {$[6,1]$} & 1.414 & 17.99543 & {$[6,1]$} & 1.414 \\
\hline 8 & 23.29609 & {$[7,1]$} & 1.490 & 23.29609 & {$[7,1]$} & 1.490 \\
\hline 9 & 29.20266 & {$[7,2]$} & 1.640 & 29.24607 & {$[7,2]$} & 1.646 \\
\hline 10 & 35.59701 & {$[8,2]$} & 1.699 & 35.63458 & {$[8,2]$} & 1.704 \\
\hline 11 & 42.47199 & {$[8,3]$} & 1.830 & 42.50370 & {$[8,3]$} & 1.834 \\
\hline 12 & 49.89776 & {$[9,3]$} & 1.877 & 49.93147 & {$[9,3]$} & 1.882 \\
\hline 13 & 57.79314 & {$[9,4]$} & 1.992 & 57.81205 & {$[9,4]$} & 1.995 \\
\hline 14 & 66.21506 & {$[10,4]$} & 2.033 & 66.23594 & {$[10,4]$} & 2.036 \\
\hline 15 & 75.09498 & {$[10,5]$} & 2.134 & 75.10986 & {$[10,5]$} & 2.137 \\
\hline 16 & 84.44850 & {$[10,5,1]$} & 2.228 & 84.49102 & {$[10,5,1]$} & 2.234 \\
\hline 17 & 94.21966 & {$[10,6,1]$} & 2.320 & 94.23676 & {$[10,6,1]$} & 2.323 \\
\hline 18 & 104.4085 & {$[11,6,1]$} & 2.349 & 104.4258 & {$[11,6,1]$} & 2.352 \\
\hline 19 & 115.0919 & {$[12,6,1]$} & 2.377 & 115.1127 & {$[11,7,1]$} & 2.434 \\
\hline 20 & 126.1922 & {$[12,7,1]$} & 2.460 & 126.2002 & {$[12,7,1]$} & 2.461 \\
\hline 21 & 137.7733 & {$[13,7,1]$} & 2.486 & 137.7859 & {$[13,7,1]$} & 2.488 \\
\hline 22 & 149.7172 & {$[12,8,2]$} & 2.608 & 149.7743 & {$[13,8,1]$} & 2.562 \\
\hline 23 & 162.0612 & {$[13,8,2]$} & 2.632 & 162.1328 & {$[13,8,2]$} & 2.635 \\
\hline 24 & 174.7901 & {$[13,8,3]$} & 2.701 & 174.8619 & {$[13,8,3]$} & 2.705 \\
\hline 25 & 187.9243 & {$[13,9,3]$} & 2.768 & 187.9888 & {$[13,9,3]$} & 2.771 \\
\hline 26 & 201.4657 & {$[14,9,3]$} & 2.788 & 201.5293 & {$[14,9,3]$} & 2.791 \\
\hline 27 & 215.3890 & {$[14,9,4]$} & 2.852 & 215.4374 & {$[14,9,4]$} & 2.855 \\
\hline 28 & 229.6947 & {$[14,10,4]$} & 2.913 & 229.7357 & {$[14,10,4]$} & 2.915 \\
\hline 29 & 244.3994 & {$[15,10,4]$} & 2.931 & 244.4401 & {$[15,10,4]$} & 2.933 \\
\hline 30 & 259.4762 & {$[15,10,5]$} & 2.990 & 259.5184 & {$[15,10,5]$} & 2.992 \\
\hline 31 & 274.9301 & {$[15,11,5]$} & 3.046 & 274.9566 & {$[15,11,5]$} & 3.048 \\
\hline 32 & 290.7250 & {$[15,11,5,1]$} & 3.101 & 290.7905 & {$[16,11,5]$} & 3.064 \\
\hline 33 & 306.8574 & {$[15,11,6,1]$} & 3.156 & 306.9034 & {$[15,11,6,1]$} & 3.159 \\
\hline
\end{tabular}




\begin{tabular}{|c|c|c|c|c|c|c|}
\hline \multicolumn{7}{|c|}{ Results for $34 \leq n \leq 77$} \\
\hline$n$ & $E_{\mathrm{MD}}$ & Configuration & $R_{\text {avg }}$ & $E_{\mathrm{CM}}$ & Configuration & $R_{\text {ext }}$ \\
\hline 34 & 323.3609 & {$[15,12,6,1]$} & 3.206 & 323.4146 & {$[16,11,6,1]$} & 3.173 \\
\hline 35 & 340.2139 & {$[16,12,6,1]$} & 3.221 & 340.2722 & {$[16,12,6,1]$} & 3.223 \\
\hline 36 & 357.4740 & {$[17,12,6,1]$} & 3.235 & 357.5053 & {$[16,12,7,1]$} & 3.274 \\
\hline 37 & 375.0712 & {$[17,12,7,1]$} & 3.286 & 375.0966 & {$[17,12,7,1]$} & 3.288 \\
\hline 38 & 393.0063 & {$[17,13,7,1]$} & 3.334 & 393.0316 & {$[17,13,7,1]$} & 3.335 \\
\hline 39 & 411.3178 & {$[17,13,7,2]$} & 3.379 & 411.3570 & {$[18,13,7,1]$} & 3.348 \\
\hline 40 & 429.9292 & {$[17,13,8,2]$} & 3.428 & 430.0216 & {$[18,13,8,1]$} & 3.396 \\
\hline 41 & 448.8795 & {$[17,14,8,2]$} & 3.470 & 449.0085 & {$[18,13,8,2]$} & 3.443 \\
\hline 42 & 468.1613 & {$[17,14,8,3]$} & 3.517 & 468.2858 & {$[17,14,8,3]$} & 3.521 \\
\hline 43 & 487.7576 & {$[17,14,9,3]$} & 3.560 & 487.8788 & {$[17,14,9,3]$} & 3.566 \\
\hline 44 & 507.6915 & {$[18,14,9,3]$} & 3.573 & 507.7930 & {$[18,14,9,3]$} & 3.576 \\
\hline 45 & 527.9573 & {$[18,15,9,3]$} & 3.613 & 528.0866 & {$[18,15,9,3]$} & 3.617 \\
\hline 46 & 548.5782 & {$[19,15,9,3]$} & 3.624 & 548.6758 & {$[18,15,9,4]$} & 3.660 \\
\hline 47 & 569.4976 & {$[18,15,10,4]$} & 3.697 & 569.5784 & {$[18,15,10,4]$} & 3.701 \\
\hline 48 & 590.7302 & {$[19,15,10,4]$} & 3.708 & 590.8007 & {$[19,15,10,4]$} & 3.710 \\
\hline 49 & 612.3010 & {$[19,16,10,4]$} & 3.746 & 612.3879 & {$[19,16,10,4]$} & 3.749 \\
\hline 50 & 634.2032 & {$[20,16,10,4]$} & 3.756 & 634.2870 & {$[20,16,10,4]$} & 3.758 \\
\hline 51 & 656.3994 & {$[19,16,11,5]$} & 3.822 & 656.4737 & {$[19,16,11,5]$} & 3.828 \\
\hline 52 & 678.9044 & {$[19,15,11,6,1]$} & 3.858 & 678.9715 & {$[20,16,11,5]$} & 3.837 \\
\hline 53 & 701.7045 & {$[18,17,11,6,1]$} & 3.920 & 701.8207 & {$[19,16,11,6,1]$} & 3.906 \\
\hline 54 & 724.7968 & {$[18,17,12,6,1]$} & 3.957 & 724.9130 & {$[20,16,11,6,1]$} & 3.913 \\
\hline 55 & 748.2062 & {$[19,17,12,6,1]$} & 3.969 & 748.3280 & {$[20,17,11,6,1]$} & 3.949 \\
\hline 56 & 771.9241 & {$[20,17,12,6,1]$} & 3.981 & 772.0267 & {$[20,17,12,6,1]$} & 3.985 \\
\hline 57 & 795.9622 & {$[20,18,12,6,1]$} & 4.014 & 796.0610 & {$[21,17,12,6,1]$} & 3.992 \\
\hline 58 & 820.3013 & {$[21,18,12,6,1]$} & 4.023 & 820.4069 & {$[21,17,12,7,1]$} & 4.028 \\
\hline 59 & 844.9824 & {$[22,18,12,6,1]$} & 4.031 & 845.0472 & {$[21,18,12,7,1]$} & 4.062 \\
\hline 60 & 869.9167 & {$[21,18,13,7,1]$} & 4.094 & 869.9729 & {$[21,18,13,7,1]$} & 4.096 \\
\hline 61 & 895.1684 & {$[22,18,13,7,1]$} & 4.101 & 895.2196 & {$[22,18,13,7,1]$} & 4.103 \\
\hline 62 & 920.7048 & {$[21,17,14,8,2]$} & 4.155 & 920.7905 & {$[22,19,13,7,1]$} & 4.136 \\
\hline 63 & 946.5161 & {$[20,19,14,8,2]$} & 4.212 & 946.6672 & {$[22,19,13,8,1]$} & 4.170 \\
\hline 64 & 972.6175 & {$[21,19,14,8,2]$} & 4.222 & 972.7978 & {$[22,19,14,8,1]$} & 4.203 \\
\hline 65 & 999.0138 & {$[20,19,14,9,3]$} & 4.270 & 999.2149 & {$[22,19,14,8,2]$} & 4.236 \\
\hline 66 & 1025.691 & {$[20,20,14,9,3]$} & 4.305 & 1025.899 & {$[22,19,14,8,3]$} & 4.269 \\
\hline 67 & 1052.661 & {$[20,20,15,9,3]$} & 4.338 & 1052.847 & {$[22,19,14,9,3]$} & 4.301 \\
\hline 68 & 1079.908 & {$[21,20,15,9,3]$} & 4.345 & 1080.113 & {$[23,19,14,9,3]$} & 4.306 \\
\hline 69 & 1107.457 & {$[22,20,15,9,3]$} & 4.354 & 1107.656 & {$[23,19,15,9,3]$} & 4.338 \\
\hline 70 & 1135.298 & {$[23,20,15,9,3]$} & 4.363 & 1135.474 & {$[23,20,15,9,3]$} & 4.367 \\
\hline 71 & 1163.410 & {$[21,21,15,10,4]$} & 4.429 & 1163.579 & {$[23,20,15,9,4]$} & 4.398 \\
\hline 72 & 1191.798 & {$[21,21,16,10,4]$} & 4.465 & 1191.945 & {$[23,20,15,10,4]$} & 4.429 \\
\hline 73 & 1220.463 & {$[22,21,16,10,4]$} & 4.472 & 1220.627 & {$[23,20,16,10,4]$} & 4.459 \\
\hline 74 & 1249.421 & {$[23,21,16,10,4]$} & 4.481 & 1249.580 & {$[24,20,16,10,4]$} & 4.464 \\
\hline 75 & 1278.653 & {$[23,21,14,11,5,1]$} & 4.503 & 1278.801 & {$[24,21,16,10,4]$} & 4.492 \\
\hline 76 & 1308.157 & {$[22,21,16,11,5,1]$} & 4.554 & 1308.330 & {$[24,21,16,10,5]$} & 4.521 \\
\hline 77 & 1337.925 & {$[22,22,16,11,5,1]$} & 4.583 & 1338.091 & {$[24,21,16,11,5]$} & 4.550 \\
\hline
\end{tabular}


Results for $78 \leq n \leq 119$

\begin{tabular}{|c|c|c|c|c|c|c|}
\hline$n$ & $E_{\mathrm{MD}}$ & Configuration & $R_{\text {avg }}$ & $E_{\mathrm{CM}}$ & Configuration & $R_{\text {ext }}$ \\
\hline 78 & 1367.963 & {$[22,22,17,11,5,1]$} & 4.612 & 1368.158 & {$[24,21,17,11,5]$} & 4.579 \\
\hline 79 & 1398.252 & {$[22,22,17,11,6,1]$} & 4.640 & 1398.493 & {$[24,22,17,11,5]$} & 4.606 \\
\hline 80 & 1428.827 & {$[22,22,17,12,6,1]$} & 4.669 & 1429.075 & {$[24,21,17,11,6,1]$} & 4.636 \\
\hline 81 & 1459.672 & {$[22,22,18,12,6,1]$} & 4.696 & 1459.914 & {$[24,21,17,12,6,1]$} & 4.664 \\
\hline 82 & 1490.795 & {$[23,22,18,12,6,1]$} & 4.702 & 1491.015 & {$[24,22,17,12,6,1]$} & 4.690 \\
\hline 83 & 1522.175 & {$[23,23,18,12,6,1]$} & 4.727 & 1522.396 & {$[25,22,17,12,6,1]$} & 4.694 \\
\hline 84 & 1553.845 & {$[24,23,18,12,6,1]$} & 4.733 & 1554.064 & {$[25,22,18,12$} & 4.721 \\
\hline 85 & 1585.785 & {$[24,24,18,12,6,1]$} & 4.757 & 1586.000 & {$[25,22,18,12,7,1]$} & 4.748 \\
\hline 86 & 1618.019 & {$[25,24,18,12,6,1]$} & 4.764 & 1618.192 & {$[25,23,18,12,7,1]$} & 4.773 \\
\hline 87 & 1650.497 & {$[24,24,18,13,7,1]$} & 4.812 & 1650.637 & {$[25,23,18,13,7,1]$} & 4.800 \\
\hline 88 & 1683.217 & {$[24,24,19,13,7,1]$} & 4.837 & 1683.354 & {$[26,23,18,13,7,1]$} & 4.803 \\
\hline 89 & 1716.199 & {$[24,24,19,13,7,2]$} & 4.860 & 1716.355 & {$[26,23,19,13,7,1]$} & 4.829 \\
\hline 90 & 1749.437 & {$[24,24,32,6,2,2]$} & 4.888 & 1749.623 & {$[26,24,19,13,7,1]$} & 4.853 \\
\hline 91 & 1782.929 & {$[24,24,19,14,8,2]$} & 4.912 & 1783.153 & {$[27,24,19,13,7,1]$} & 4.857 \\
\hline 92 & 1816.674 & {$[24,24,19,14,8,3]$} & 4.934 & 1816 & $9,14,7,1]$ & 4.883 \\
\hline 93 & 1850.697 & {$[24,24,19,14,9,3]$} & 4.957 & 1850.998 & {$[27,24,19,14,8,1]$} & 4.908 \\
\hline 94 & 1884.964 & {$[24,24,20,14,9,3]$} & 4.984 & 1885.300 & {$[27,24,19,14,8,2]$} & 4.933 \\
\hline 95 & 1919.486 & {$[25,25,19,14,9,3]$} & 4.990 & 1919.823 & {$[26,24,19,14,9,3]$} & 4.981 \\
\hline 96 & 1954.254 & {$[25,25,20,14,9,3]$} & 5.013 & 1954.592 & {$[26,24,20,14$} & 5.005 \\
\hline 97 & 1989.285 & {$[25,25$} & 5.038 & 1989 & {$[27,24,20,14$} & 5.008 \\
\hline 98 & 2024.569 & {$[25,25,21,15,9,3]$} & 5.061 & 2024.897 & {$[27,24,20,15,9,3]$} & 5.032 \\
\hline 99 & 2060.131 & {$[26,25,21,15,9,3]$} & 5.066 & 2060.434 & {$[27,25,20,15,9,3]$} & 5.055 \\
\hline 100 & 2095.931 & {$[26,26,21,15,9,3]$} & 5.088 & 2096.242 & {$[27,25,20,15,9,4]$} & 5.079 \\
\hline 101 & 2131.979 & {$[26,26,21,14,10,4]$} & 5.112 & 2132.277 & {$[27,25,20,15,10,4]$} & 5.103 \\
\hline 102 & 2168.273 & {$[26,26,20,16,10,4]$} & 5.136 & 2168.562 & {$[27,25,21,15,10,4]$} & 5.126 \\
\hline 103 & 2204.812 & {$[26,26,21,16,10,4]$} & 5.158 & 2205.103 & {$[27,25,21,16,10,4]$} & 5.149 \\
\hline 104 & 2241.601 & {$[26,26,22,16,10,4]$} & 5.180 & 2241.889 & {$[28,25,21,16,10,4]$} & 5.152 \\
\hline 105 & 2278.670 & {$[27,26,22,16,10,4]$} & 5.185 & 2278.931 & {$[28,26,21,16,10,4]$} & 5.174 \\
\hline 106 & 2315.960 & {$[26,26,21,16,11,5,1]$} & 5.222 & 2316.247 & {$[29,26,21,16,10,4]$} & 5.176 \\
\hline 107 & 2353.486 & {$[26,26,22,15,11,6,1]$} & 5.249 & 2353.799 & {$[28,26,21,16,11,5]$} & 5.220 \\
\hline 108 & 2391.253 & {$[26,26,20,17,12,6,1]$} & 5.273 & 2391.574 & {$[28,26,22,16,11,5]$} & 5.242 \\
\hline 109 & 2429.264 & {$[26,26,21,17,12,6,1]$} & 5.294 & 2429.600 & {$[28,26,22,17,11,5]$} & 5.265 \\
\hline 110 & 2467.525 & {$[26,26,21,18,12,6,1]$} & 5.315 & 2467.872 & {$[29,26,22,17,11,5]$} & 5.267 \\
\hline 111 & 2506.023 & {$[27,27,20,18,12,6,1]$} & 5.320 & 2506.392 & {$[29,27,22,17,11,5]$} & 5.288 \\
\hline 112 & 2544.754 & {$[27,27,21,18,12,6,1]$} & 5.341 & 2545.141 & {$[28,26,22,17,12,6,1]$} & 5.332 \\
\hline 113 & 2583.739 & {$[27,27,22,18,12,6,1]$} & 5.361 & 2584.123 & {$[28,27,22,17,12,6,1]$} & 5.352 \\
\hline 114 & 2622.965 & {$[27,27,23,18,12,6,1]$} & 5.382 & 2623.339 & {$[29,27,22,17,12,6,1]$} & 5.354 \\
\hline 115 & 2662.432 & {$[27,27,24,18,12,6,1]$} & 5.402 & 2662.804 & {$[29,27,23,17,12,6,1]$} & 5.375 \\
\hline 116 & 2702.166 & {$[28,28,23,18,12,6,1]$} & 5.406 & 2702.508 & {$[29,27,23,18,12,6,1]$} & 5.397 \\
\hline 117 & 2742.111 & {$[28,28,24,18,12,6,1]$} & 5.426 & 2742.473 & {$[30,27,23,18,12,6,1]$} & 5.399 \\
\hline 118 & 2782.346 & {$[29,28,24,18,12,6,1]$} & 5.430 & 2782.676 & {$[30,28,23,18,12,6,1]$} & 5.419 \\
\hline 119 & 2822.794 & {$[29,29,24,18,12,6,1]$} & 5.449 & 2823.104 & {$[29,28,23,18,13,7,1]$} & 5.459 \\
\hline
\end{tabular}


Results for $120 \leq n \leq 160$

\begin{tabular}{|c|c|c|c|c|c|c|}
\hline$n$ & $E_{\mathrm{MD}}$ & Configuration & $R_{\text {avg }}$ & $E_{\mathrm{CM}}$ & Configuration & $R_{\text {ext }}$ \\
\hline 120 & 2863.472 & {$[28,28,24,19,12,8,1]$} & 5.489 & 2863.765 & {$[30,28,23,18,13,7,1]$} & 5.461 \\
\hline 121 & 2904.384 & {$[28,28,41,14,6,2,2]$} & 5.511 & 2904.669 & {$[30,28,24,18,13,7,1]$} & 5.482 \\
\hline 122 & 2945.520 & {$[28,28,25,17,14,8,2]$} & 5.531 & 2945.811 & {$[30,28,24,19,13,7,1]$} & 5.502 \\
\hline 123 & 2986.892 & {$[28,28,25,32,6,2,2]$} & 5.550 & 2987.209 & {$[31,28,24,19,13,7,1]$} & 5.504 \\
\hline 124 & 3028.501 & {$[28,28,42,14,6,3,3]$} & 5.571 & 3028.839 & {$[31,29,24,19,13,7,1]$} & 5.524 \\
\hline 125 & 3070.333 & {$[29,29,25,16,14,9,3]$} & 5.574 & 3070.723 & {$[31,29,25,19,13,7,1]$} & 5.544 \\
\hline 126 & 3112.398 & {$[29,29,22,20,14,9,3]$} & 5.594 & 3112.844 & {$[31,29,25,19,14,7,1]$} & 5.564 \\
\hline 127 & 3154.706 & {$[29,29,25,18,14,9,3]$} & 5.613 & 3155.173 & {$[31,29,25,19,14,8,1]$} & 5.584 \\
\hline 128 & 3197.232 & {$[29,29,23,20,15,9,3]$} & 5.633 & 3197.732 & {$[31,29,25,20,14,8,1]$} & 5.604 \\
\hline 129 & 3240.000 & {$[29,29,24,20,15,9,3]$} & 5.652 & 3240.519 & {$[31,29,25,20,14,8,2]$} & 5.625 \\
\hline 130 & 3282.996 & {$[29,29,24,21,15,9,3]$} & 5.671 & 3283.503 & {$[30,29,25,20,14,9,3]$} & 5.663 \\
\hline 131 & 3326.229 & {$[29,29,25,21,15,9,3]$} & 5.690 & 3326.718 & {$[31,29,25,20,14,9,3]$} & 5.664 \\
\hline 132 & 3369.693 & {$[29,29,26,21,15,9,3]$} & 5.708 & 3370.165 & {$[31,29,25,20,15,9,3]$} & 5.684 \\
\hline 133 & 3413.392 & {$[30,30,26,20,15,9,3]$} & 5.711 & 3413.863 & {$[31,30,25,20,15,9,3]$} & 5.702 \\
\hline 134 & 3457.312 & {$[30,30,26,21,15,9,3]$} & 5.730 & 3457.790 & {$[31,30,26,20,15,9,3]$} & 5.721 \\
\hline 135 & 3501.457 & {$[30,30,24,21,16,10,4]$} & 5.750 & 3501.939 & {$[31,30,26,21,15,9,3]$} & 5.741 \\
\hline 136 & 3545.825 & {$[30,30,27,19,16,10,4]$} & 5.768 & 3546.313 & {$[31,29,26,21,15,10,4]$} & 5.761 \\
\hline 137 & 3590.431 & {$[30,30,27,20,16,10,4]$} & 5.786 & 3590.891 & {$[31,30,26,21,15,10,4]$} & 5.779 \\
\hline 138 & 3635.262 & {$[30,30,26,22,16,10,4]$} & 5.805 & 3635.710 & {$[31,30,26,21,16,10,4]$} & 5.798 \\
\hline 139 & 3680.315 & {$[30,30,27,22,16,10,4]$} & 5.822 & 3680.751 & {$[32,30,26,21,16,10,4]$} & 5.799 \\
\hline 140 & 3725.602 & {$[30,30,28,22,16,10,4]$} & 5.840 & 3726.043 & {$[32,31,26,21,16,10,4]$} & 5.817 \\
\hline 141 & 3771.105 & {$[30,30,2,44,17,11,6,1]$} & 5.861 & 3771.558 & {$[32,31,27,21,16,10,4]$} & 5.835 \\
\hline 142 & 3816.828 & {$[31,31,23,22,17,11,6,1]$} & 5.864 & 3817.291 & {$[32,31,27,22,16,10,4]$} & 5.854 \\
\hline 143 & 3862.771 & {$[30,30,26,22,17,11,6,1]$} & 5.896 & 3863.253 & {$[33,31,27,22,16,10,4]$} & 5.855 \\
\hline 144 & 3908.934 & {$[31,31,25,22,17,11,6,1]$} & 5.899 & 3909.439 & {$[32,31,27,22,16,11,5]$} & 5.891 \\
\hline 145 & 3955.315 & {$[30,30,26,23,17,12,6,1]$} & 5.932 & 3955.833 & {$[32,31,27,22,17,11,5]$} & 5.909 \\
\hline 146 & 4001.923 & {$[31,31,25,23,17,12,6,1]$} & 5.936 & 4002.450 & {$[33,31,27,22,17,11,5]$} & 5.910 \\
\hline 147 & 4048.753 & {$[31,31,25,23,18,12,6,1]$} & 5.953 & 4049.312 & {$[33,32,27,22,17,11,5]$} & 5.927 \\
\hline 148 & 4095.803 & {$[30,30,28,23,18,12,6,1]$} & 5.984 & 4096.391 & {$[33,32,28,22,17,11,5]$} & 5.945 \\
\hline 149 & 4143.077 & {$[31,31,26,24,18,12,6,1]$} & 5.989 & 4143.661 & {$[32,31,27,23,17,12,6,1]$} & 5.982 \\
\hline 150 & 4190.571 & {$[30,30,29,24,18,12,6,1]$} & 6.019 & 4191.146 & {$[33,31,27,23,17,12,6,1]$} & 5.983 \\
\hline 151 & 4238.292 & {$[31,31,28,24,18,12,6,1]$} & 6.022 & 4238.852 & {$[33,31,28,23,17,12,6,1]$} & 6.000 \\
\hline 152 & 4286.238 & {$[32,32,27,24,18,12,6,1]$} & 6.025 & 4286.779 & {$[33,32,28,23,17,12,6,1]$} & 6.016 \\
\hline 153 & 4334.404 & {$[32,32,28,24,18,12,6,1]$} & 6.041 & 4334.920 & {$[33,32,28,23,18,12,6,1]$} & 6.034 \\
\hline 154 & 4382.789 & {$[32,32,29,24,18,12,6,1]$} & 6.058 & 4383.304 & {$[34,32,28,23,18,12,6,1]$} & 6.035 \\
\hline 155 & 4431.402 & {$[32,32,30,24,18,12,6,1]$} & 6.074 & 4431.919 & {$[34,32,28,24,18,12,6,1]$} & 6.052 \\
\hline 156 & 4480.240 & {$[33,33,29,24,18,12,6,1]$} & 6.076 & 4480.739 & {$[33,32,28,24,18,13,7,1]$} & 6.087 \\
\hline 157 & 4529.280 & {$[33,33,30,24,18,12,6,1]$} & 6.092 & 4529.763 & {$[34,32,28,24,18,13,7,1]$} & 6.087 \\
\hline 158 & 4578.533 & {$[31,31,53,19,14,6,2,2]$} & 6.140 & 4579.002 & {$[34,32,29,24,18,13,7,1]$} & 6.104 \\
\hline 159 & 4627.983 & {$[32,32,30,41,14,6,2,2]$} & 6.144 & 4628.461 & {$[34,33,29,24,18,13,7,1]$} & 6.120 \\
\hline 160 & 4677.653 & {$[32,32,53,19,14,6,2,2]$} & 6.159 & 4678.133 & {$[34,33,29,24,19,13,7,1]$} & 6.137 \\
\hline
\end{tabular}


Results for $161 \leq n \leq 200$

\begin{tabular}{|c|c|c|c|c|c|c|}
\hline$n$ & $E_{\mathrm{MD}}$ & Configuration & $R_{\text {avg }}$ & $E_{\mathrm{CM}}$ & Configuration & $R_{\text {ext }}$ \\
\hline 161 & 4727.551 & {$[33,33,30,41,14,6,2,2]$} & 6.163 & 4728.043 & {$[35,33,29,24,19,13,7,1]$} & 6.138 \\
\hline 162 & 4777.653 & {$[32,32,54,20,14,6,2,2]$} & 6.192 & 4778.179 & {$[35,33,29,25,19,13,7,1]$} & 6.155 \\
\hline 163 & 4827.973 & {$[32,32,55,20,14,6,2,2]$} & 6.208 & 4828.523 & {$[35,33,30,25,19,13,7,1]$} & 6.171 \\
\hline 164 & 4878.503 & {$[31,31,31,25,20,14,9,3]$} & 6.237 & 4879.078 & {$[35,34,30,25,19,13,7,1]$} & 6.187 \\
\hline 165 & 4929.243 & {$[32,32,55,20,14,6,3,3]$} & 6.241 & 4929.869 & {$[36,34,30,25,19,13,7,1]$} & 6.188 \\
\hline 166 & 4980.198 & {$[31,31,31,25,21,15,9,3]$} & 6.270 & 4980.862 & {$[35,34,30,25,19,14,8,1]$} & 6.221 \\
\hline 167 & 5031.363 & {$[32,32,31,45,15,6,3,3]$} & 6.274 & 5032.046 & {$[35,34,30,25,20,14,8,1]$} & 6.237 \\
\hline 168 & 5082.740 & {$[32,32,31,26,20,15,9,3]$} & 6.288 & 5083.459 & {$[35,34,30,25,20,14,8,2]$} & 6.254 \\
\hline 169 & 5134.328 & {$[32,32,31,26,21,15,9,3]$} & 6.305 & 5135.059 & {$[34,34,30,25,20,14,9,3]$} & 6.287 \\
\hline 170 & 5186.133 & {$[32,32,32,26,21,15,9,3]$} & 6.320 & 5186.850 & {$[35,34,30,25,20,14,9,3]$} & 6.287 \\
\hline 171 & 5238.158 & {$[33,33,57,21,15,6,3,3]$} & 6.323 & 5238.859 & {$[35,34,30,25,20,15,9,3]$} & 6.303 \\
\hline 172 & 5290.383 & {$[33,33,31,27,21,15,9,3]$} & 6.338 & 5291.081 & {$[35,34,30,26,20,15,9,3]$} & 6.319 \\
\hline 173 & 5342.830 & {$[33,33,32,27,21,15,9,3]$} & 6.354 & 5343.522 & {$[35,34,31,26,20,15,9,3]$} & 6.335 \\
\hline 174 & 5395.482 & {$[32,32,32,26,22,16,10,4]$} & 6.382 & 5396.169 & {$[35,34,31,26,21,15,9,3]$} & 6.351 \\
\hline 175 & 5448.340 & {$[33,64,26,22,16,10,2,2]$} & 6.385 & 5449.031 & {$[36,34,31,26,21,15,9,3]$} & 6.352 \\
\hline 176 & 5501.406 & {$[33,33,32,27,21,16,10,4]$} & 6.402 & 5502.099 & {$[36,35,31,26,21,15,9,3]$} & 6.366 \\
\hline 177 & 5554.678 & {$[33,33,32,27,22,16,10,4]$} & 6.416 & 5555. & {$[35,35,31,26,21,15,10,4]$} & 6.398 \\
\hline 178 & 5608.165 & {$[33,33,33,27,22,16,10,4]$} & 6.431 & 5608.845 & {$[36,35,31,26,21,15,10,4]$} & 6.398 \\
\hline 179 & 5661.871 & {$[34,34,59,22,16,10,2,2]$} & 6.434 & 5662.526 & {$[36,35,31,26,21,16,10,4]$} & 6.414 \\
\hline 180 & 5715.769 & {$[34,34,32,28,22,16,10,4]$} & 6.449 & 5716.419 & {$[36,35,31,27,21,16,10,4]$} & 6.430 \\
\hline 181 & 5769.872 & {$[33,33,2,31,47,17,11,6,1]$} & 6.480 & 5770.531 & {$[36,35,32,27,21,16,10,4]$} & 6.445 \\
\hline 182 & 5824.177 & {$[33,33,33,28,20,17,11,6,1]$} & 6.494 & 5824.842 & {$[36,35,32,27,22,16,10,4]$} & 6.461 \\
\hline 183 & 5878.689 & {$[33,33,60,5,17,17,11,6,1]$} & 6.506 & 5879.372 & {$[36,36,32,27,22,16,10,4]$} & 6.475 \\
\hline 184 & 5933.401 & {$[34,34,34,28,18,17,12,6,1]$} & 6.514 & 5934.105 & {$[37,36,32,27,22,16,10,4]$} & 6.475 \\
\hline 185 & 5988.323 & {$[34,34,1,5$} & 6.527 & 5989.067 & {$[36,36,32,27,22,16,11,5]$} & 6.506 \\
\hline 186 & 6043.450 & {$[34,34,34,25,23,17,12,6,1]$} & 6.542 & 6044.200 & {$[36,36,32,27,22,17,11,5]$} & 6.522 \\
\hline 187 & 6098.766 & {$[34,34,34,24,24,18,12,6,1]$} & 6.559 & 6099.538 & {$[37,36,32,27,22,17,11,5]$} & 6.522 \\
\hline 188 & 6154.300 & {$[34,34,34,25,24,18,12,6,1]$} & 6.572 & 6155.080 & {$[37,36,32,28,22,17,11,5]$} & 6.537 \\
\hline 189 & 6210.038 & {$[34,34,34,26,24,18,12,6,1]$} & 6.586 & 6210.839 & {$[37,36,32,28,23,17,11,5]$} & 6.552 \\
\hline 190 & 6265.982 & {$[34,34,34,27,24,18,12,6,1]$} & 6.600 & 6266.794 & {$[37,36,33,28,23,17,11,5]$} & 6.567 \\
\hline 191 & 6322.128 & {$[34,34,34,28,24,18,12,6,1]$} & 6.614 & 6322.955 & {$[36,36,32,28,23,17,12,6,1]$} & 6.598 \\
\hline 192 & 6378.489 & {$[34,34,34,29,24,18,12,6,1]$} & 6.628 & 6379.295 & {$[37,36,32,28,23,17,12,6,1]$} & 6.597 \\
\hline 193 & 6435.051 & {$[35,35,34,28,24,18,12,6,1]$} & 6.630 & 6435.837 & {$[37,36,33,28,23,17,12,6,1]$} & 6.612 \\
\hline 194 & 6491.807 & {$[35,35,34,29,24,18,12,6,1]$} & 6.644 & 6492.585 & {$[37,36,33,28,23,18,12,6,1]$} & 6.627 \\
\hline 195 & 6548.773 & {$[35,35,35,29,24,18,12,6,1]$} & 6.658 & 6549.548 & {$[37,37,33,28,23,18,12,6,1]$} & 6.641 \\
\hline 196 & 6605.955 & {$[35,35,35,30,24,18,12,6,1]$} & 6.672 & 6606.711 & {$[37,37,33,29,23,18,12,6,1]$} & 6.655 \\
\hline 197 & 6663.328 & {$[36,36,34,30,24,18,12,6,1]$} & 6.674 & 6664.081 & {$[38,37,33,29,23,18,12,6,1]$} & 6.655 \\
\hline 198 & 6720.909 & {$[36,36,35,30,24,18,12,6,1]$} & 6.688 & 6721.650 & {$[38,37,33,29,24,18,12,6,1]$} & 6.670 \\
\hline 199 & 6778.722 & {$[35,1,35,35,30,44,12,6,1]$} & 6.710 & 6779.422 & {$[38,37,34,29,24,18,12,6,1]$} & 6.684 \\
\hline 200 & 6836.692 & {$[35,35,35,53,19,13,6,2,2]$} & 6.729 & 6837.393 & {$[37,37,34,29,24,18,13,7,1]$} & 6.714 \\
\hline
\end{tabular}

